MATHEMATICS OF COMPUTATION

Volume 74, Number 252, Pages 1575-1598

S 0025-5718(05)01759-X

Article electronically published on May 5, 2005

\title{
THE APPROXIMATION \\ OF THE MAXWELL EIGENVALUE PROBLEM USING A LEAST-SQUARES METHOD
}

\author{
JAMES H. BRAMBLE, TZANIO V. KOLEV, AND JOSEPH E. PASCIAK
}

\begin{abstract}
In this paper we consider an approximation to the Maxwell's eigenvalue problem based on a very weak formulation of two div-curl systems with complementary boundary conditions. We formulate each of these div-curl systems as a general variational problem with different test and trial spaces, i.e., the solution space is $\boldsymbol{L}^{2}(\Omega) \equiv\left(L^{2}(\Omega)\right)^{3}$ and components in the test spaces are in subspaces of $H^{1}(\Omega)$, the Sobolev space of order one on the computational domain $\Omega$. A finite-element least-squares approximation to these variational problems is used as a basis for the approximation. Using the structure of the continuous eigenvalue problem, a discrete approximation to the eigenvalues is set up involving only the approximation to either of the div-curl systems. We give some theorems that guarantee the convergence of the eigenvalues to those of the continuous problem without the occurrence of spurious values. Finally, some results of numerical experiments are given.
\end{abstract}

\section{INTRODUCTION}

In this paper we consider the eigenvalue problem associated with Maxwell's equations. These equations can, for example, be used to determine the frequencies that will propagate through a medium such as a waveguide 3, 32. It looks promising that similar ideas can be applied to the more general equations describing propagation through photonic crystals; see [23, 38].

Although two dimensional versions of Maxwell's eigenvalue problem often result in eigenvalue problems involving the Laplacian, three dimensional problems are significantly more complicated as they result in an eigenvalue problem involving curl-curl, an operator that is not elliptic. Accordingly, the inverse is no longer compact which leads to a much more complicated analysis. However, as we shall see, a compact "pseudo" inverse can be constructed that has the same eigenvectors.

One of the more popular approaches for approximating Maxwell's eigenvalue problem is based on using curl-conforming spaces such as those developed by Nédélec (cf. [34, 35]). In such a method one looks for solutions to the problem in $\boldsymbol{H}$ (curl), the space of vector functions which, along with their curls, are

Received by the editor April 23, 2004 and, in revised form, October 12, 2004.

2000 Mathematics Subject Classification. 65F10, 65N30.

Key words and phrases. Maxwell eigenvalues, div-curl systems,inf-sup condition, finite element approximation, negative norm least-squares, Maxwell's equations.

This work is based upon work supported by the National Science Foundation under grant No. 0311902. 
in $\boldsymbol{L}^{2}(\Omega)$. Analysis of the eigenvalue problem using these spaces either involves proving collective compactness [29, 33] or proving convergence in norm [4, 5, 6, 22].

Early engineering approximations to these equations were often attempted using $H^{1}$-conforming finite-element spaces [7. These were known to have problems due to low regularity solutions and multiple valued potentials [19, 24, 30. Recently, new methods for dealing with these problems have been proposed [13, 14, 36]. The methods of 14 depend on weighted functionals with weights depending on the strength of the singularities at corners and edges. In [36], discontinuous Galerkin methods are proposed.

The approach we take in this paper is to first relate the problem to a block system involving the solution of div-curl systems. These div-curl systems are formulated as variational problems following [12] in which the solutions are posed in $\boldsymbol{L}^{2}(\Omega)$ and the (components of the) test functions are in various subspaces of the Sobolev space $H^{1}(\Omega)$. This results in a very weak formulation of the div-curl problem where the data can reside in a negative norm space, e.g., in the dual of the test spaces. That the test functions are in $H^{1}(\Omega)$ is a critical attribute of the method we take advantage of in our subsequent analysis of the Maxwell eigenvalue problem. Indeed, this leads to solution operators for the div-curl problem that are bounded from $H^{-1}(\Omega)$ into $L^{2}(\Omega)$ in the continuous as well as the discrete case. Since the approximation is based in $\boldsymbol{L}^{2}(\Omega)$, our approximation subspaces can be very simple. For example, we can use discontinuous functions at the material interfaces where the solutions jump while using $C^{0}$ elements in the interior where the solution is smooth.

In this paper we shall show how our variational form of the div-curl system can be used to develop a stable approximation to the Maxwell eigenvalue problem. We first show that the eigenfunctions with nonzero eigenvalues are also eigenfunctions of a block-compact skew-Hermitian problem where the blocks correspond to div-curl problems. We use our div-curl approximation to derive a sequence of approximation operators which converge in norm to the above-mentioned compact operator.

Actual three-dimensional applications necessarily contain large numbers of unknowns (on the order of millions). Such a large number of unknowns result from complicated device geometry and the mesh refinement necessary for resolving singular behavior in the solutions. Since the systems are too large for conventional direct eigensolvers, the eigenvalues must be computed iteratively. To obtain a system which is more amenable to iterative computation, we show that the original eigenpairs can be computed from those of a compact symmetric real operator. This system can be approximated in norm by the discrete operator for one div-curl system and its adjoint, and it results in a symmetric discrete eigenvalue problem. The development of effective iterative techniques for computing the eigenvalues of large symmetric problems has been the subject of intensive research in the past two decades; e.g., [18, 26, 10. These methods are more efficient and robust than those developed for nonsymmetric and/or indefinite systems. Thus, the reformulation of the problem as a symmetric real system represents a significant computational advantage.

The outline of the remainder of this paper is as follows. In Section 2 we describe the Maxwell eigenvalue problem and reformulate it in terms of variational divcurl systems. In Section 3 we show how the discrete technique of 12 can be used to develop approximations to the reformulated variational div-curl system. 
These operators are used to construct symmetric discrete eigenproblems in Section 4. Earlier sections of this paper are presented under the assumption of a simply connected domain with a connected boundary. We extend the techniques herein to the case of multiply connected domains with multiply connected boundaries in Section 5. Finally, the results of numerical experiments illustrating the convergence behavior of the eigenvalue approximations are given in Section 6 .

\section{The Maxwell eigenvalue Problem}

Let $\Omega$ be a polyhedral domain with Lipschitz boundary in $\mathbb{R}^{3}$. For simplicity, we shall assume that $\Omega$ is simply connected with a simply connected boundary. We shall address the case of nonsimply connected domains and domains whose boundaries consist of multiply connected components in Section 5 .

Maxwell eigenvalue problems involve the quantities $\boldsymbol{h}, \boldsymbol{e}: \Omega \rightarrow \mathbb{C}^{3}$, respectively, the magnetic and electric field intensity. The Maxwell eigenvalue problem is given by

$$
\begin{aligned}
& \nabla \times \boldsymbol{h}=\lambda \epsilon \boldsymbol{e} \quad \text { in } \Omega, \\
& \nabla \times \boldsymbol{e}=-\lambda \mu \boldsymbol{h} \text { in } \Omega, \\
& \boldsymbol{e} \times \boldsymbol{n}=\mathbf{0} \text { on } \partial \Omega .
\end{aligned}
$$

Here $\boldsymbol{n}$ denotes the outward normal on $\partial \Omega$. In addition, the real-valued functions $\mu$ and $\epsilon$ describe the experimentally determined material properties, respectively, the magnetic permeability and the electric permittivity. Typically, these are piecewise smooth functions that are bounded above and below with jumps at the material interfaces. Clearly, the above problem has a large null eigenspace made up of gradients. We are only interested in the nonzero eigenvalues $\lambda$ and the corresponding eigenfunctions $\boldsymbol{h}, \boldsymbol{e}$.

We shall use the following complex spaces, where $\gamma \in\{\epsilon, \mu\}$ :

$$
\begin{aligned}
\boldsymbol{L}_{\gamma}^{2}(\Omega) & -\boldsymbol{L}^{2}(\Omega) \text { equipped with the weighted inner product }(\boldsymbol{u}, \boldsymbol{v})_{\gamma}=(\gamma \boldsymbol{u}, \boldsymbol{v})_{\boldsymbol{L}^{2}(\Omega),}, \\
\boldsymbol{H}(\mathbf{c u r l}) & =\left\{\boldsymbol{v} \in \boldsymbol{L}^{2}(\Omega), \nabla \times \boldsymbol{v} \in \boldsymbol{L}^{2}(\Omega)\right\}, \\
\boldsymbol{H}_{0}(\mathbf{c u r l}) & =\{\boldsymbol{v} \in \boldsymbol{H}(\mathbf{c u r l}),: \boldsymbol{v} \times \boldsymbol{n}=\mathbf{0} \text { on } \partial \Omega\}, \\
\boldsymbol{H}(\operatorname{div} ; \gamma) & =\left\{\boldsymbol{v} \in \boldsymbol{L}^{2}(\Omega), \nabla \cdot(\gamma \boldsymbol{v}) \in L^{2}(\Omega)\right\}, \\
\boldsymbol{H}_{0}(\operatorname{div} ; \gamma) & =\{\boldsymbol{v} \in \boldsymbol{H}(\operatorname{div} ; \gamma): \boldsymbol{n} \cdot(\gamma \boldsymbol{v})=0 \text { on } \partial \Omega\},
\end{aligned}
$$

A more precise statement of the eigenvalue problem now reads as follows. Find $\lambda \in \mathbb{C}, \boldsymbol{h} \in \boldsymbol{H}$ (curl) and $\boldsymbol{e} \in \boldsymbol{H}_{0}$ (curl) satisfying the first two equations of (2.1).

One way to analyze the above problem is to look for $(\boldsymbol{e}, \boldsymbol{h})$ in $\boldsymbol{H}_{0}(\mathbf{c u r l}) \times \boldsymbol{H}(\mathbf{c u r l})$. In contrast, we look for solutions in $\boldsymbol{L}^{2}(\Omega) \times \boldsymbol{L}^{2}(\Omega)$. This is natural as we ultimately end up with a compact operator on $\boldsymbol{L}^{2}(\Omega) \times \boldsymbol{L}^{2}(\Omega)$.

We shall characterize the above eigenvalue problem by showing that it is related to an eigenvalue problem involving a compact Hermitian semidefinite operator. Suppose we have an eigenfunction pair, $\boldsymbol{e} \in \boldsymbol{H}_{0}$ (curl) and $\boldsymbol{h} \in \boldsymbol{H}$ (curl) with a nonzero eigenvalue $\lambda$. Then, it follows that

$$
0=(\nabla \times \boldsymbol{h}, \nabla \theta)=\lambda(\epsilon \boldsymbol{e}, \nabla \theta) \text { for all } \theta \in H_{0}^{1}(\Omega) .
$$

Note that the integration by parts formula

$$
(\nabla \times \boldsymbol{w}, \boldsymbol{v})=(\boldsymbol{w}, \nabla \times \boldsymbol{v})
$$


for all $\boldsymbol{w} \in \boldsymbol{H}\left(\right.$ curl) and $\boldsymbol{v} \in \boldsymbol{H}_{0}($ curl $)$ follows from the density of $\mathcal{D}(\Omega)^{3}$ in $\boldsymbol{H}_{0}(\mathbf{c u r l})$. Thus,

$$
0=(\nabla \times \boldsymbol{e}, \nabla \theta)=-\lambda(\mu \boldsymbol{h}, \nabla \theta) \quad \text { for all } \theta \in H^{1}(\Omega)
$$

and

$$
\boldsymbol{e} \in \boldsymbol{H}_{0}(\mathbf{c u r l}) \cap \boldsymbol{H}(\operatorname{div} ; \epsilon) \text { and } \boldsymbol{h} \in \boldsymbol{H}(\mathbf{c u r l}) \cap \boldsymbol{H}_{0}(\operatorname{div} ; \mu) .
$$

We shall assume that $\epsilon$ and $\mu$ are regular enough so that $\boldsymbol{H}_{0}(\mathbf{c u r l}) \cap \boldsymbol{H}(\operatorname{div} ; \epsilon)$ and $\boldsymbol{H}($ curl $) \cap \boldsymbol{H}_{0}($ div; $\mu)$ are continuously embedded in $\left(H^{s}(\Omega)\right)^{3}$ for some $s>0$. Such results are provided in 15 when $\epsilon$ and $\mu$ are piecewise smooth.

From the above discussion, it is natural to consider two source problems. The first is the div-curl system

$$
\begin{aligned}
\nabla \times \boldsymbol{h} & =\epsilon \boldsymbol{g}_{1} \text { in } \Omega, \\
\nabla \cdot(\mu \boldsymbol{h}) & =0 \text { in } \Omega, \\
\boldsymbol{h} \cdot \boldsymbol{n} & =0 \text { on } \partial \Omega .
\end{aligned}
$$

The second div-curl system is similar:

$$
\begin{aligned}
\nabla \times \boldsymbol{e} & =\mu \boldsymbol{g}_{2} \text { in } \Omega, \\
\nabla \cdot(\epsilon \boldsymbol{e}) & =0 \text { in } \Omega, \\
\boldsymbol{e} \times \boldsymbol{n} & =\mathbf{0} \text { on } \partial \Omega .
\end{aligned}
$$

An $\boldsymbol{L}^{2}(\Omega)$-based variational framework for the solution of the above two problems in the case of real-valued functions was given in [12. The extension of this framework to complex spaces is straightforward. To describe this formulation, we introduce the spaces

$$
\begin{aligned}
H_{1} & =H^{1}(\Omega), \quad H_{2}=H_{0}^{1}(\Omega), \\
\boldsymbol{V}_{1} & =\boldsymbol{H}_{0}^{1}(\Omega) \equiv\left(H_{0}^{1}(\Omega)\right)^{3}, \quad \boldsymbol{V}_{2}=\left(H^{1}(\Omega)\right)^{3}, \\
\boldsymbol{V}_{1,0} & =\left\{\boldsymbol{w}=\nabla \psi, \psi \in H_{0}^{2}(\Omega)\right\}, \\
\boldsymbol{V}_{2,0} & =\left\{\boldsymbol{w}=\nabla \psi, \psi \in H^{2}(\Omega)\right\} .
\end{aligned}
$$

For $k=1,2$, let $\boldsymbol{Y}_{k}=\boldsymbol{V}_{k} \times H_{k}$ and consider the sesquilinear forms on $\boldsymbol{L}^{2}(\Omega) \times \boldsymbol{Y}_{k}$ defined by

$$
b_{1}(\boldsymbol{x},(\boldsymbol{v}, h))=(\boldsymbol{x}, \nabla \times \boldsymbol{v})+(\mu \boldsymbol{x}, \nabla h) \quad \text { for all } \boldsymbol{x} \in \boldsymbol{L}^{2}(\Omega) \text { and }(\boldsymbol{v}, h) \in \boldsymbol{Y}_{1}
$$

and

$$
b_{2}(\boldsymbol{x},(\boldsymbol{v}, h))=(\boldsymbol{x}, \nabla \times \boldsymbol{v})+(\epsilon \boldsymbol{x}, \nabla h) \quad \text { for all } \boldsymbol{x} \in \boldsymbol{L}^{2}(\Omega) \text { and }(\boldsymbol{v}, h) \in \boldsymbol{Y}_{2} .
$$

It is shown in 12 that there is a unique function $\boldsymbol{x}_{k} \in \boldsymbol{L}^{2}(\Omega)$ satisfying

$$
b_{k}\left(\boldsymbol{x}_{k},(\boldsymbol{v}, h)\right)=\left\langle\boldsymbol{f}_{k}, \boldsymbol{v}\right\rangle \quad \text { for all }(\boldsymbol{v}, h) \in \boldsymbol{Y}_{k}
$$

for any functional $\boldsymbol{f}_{k} \in \boldsymbol{V}_{k}^{*}$ satisfying the compatibility condition

$$
\left\langle\boldsymbol{f}_{k}, \boldsymbol{v}\right\rangle=0 \text { for all } \boldsymbol{v} \in \boldsymbol{V}_{k, 0} .
$$

This analysis is based the inf-sup condition

$$
\left\|\boldsymbol{x}_{k}\right\|_{\boldsymbol{L}^{2}(\Omega)} \leq C \sup _{(\boldsymbol{v}, h) \in \boldsymbol{Y}_{k}} \frac{\left|b_{k}\left(\boldsymbol{x}_{k},(\boldsymbol{v}, h)\right)\right|}{\|(\boldsymbol{v}, h)\|_{\boldsymbol{Y}_{k}}} \text { for all } \boldsymbol{x}_{k} \in \boldsymbol{L}^{2}(\Omega),
$$

which was proved in 12. Here and in what follows, $C$ is used to denote a generic positive constant. 
We next consider the compatibility condition (2.8). Let $\overline{\boldsymbol{V}}_{1,0}=\{\nabla \phi: \phi \epsilon$ $\left.H_{0}^{1}(\Omega)\right\}$ and $\overline{\boldsymbol{V}}_{2,0}=\left\{\nabla \phi: \phi \in H^{1}(\Omega)\right\}$. Then $\overline{\boldsymbol{V}}_{k, 0}$ is the closure in $\boldsymbol{L}^{2}(\Omega)$ of $\boldsymbol{V}_{k, 0}$ for $k=1,2$. Let $\boldsymbol{Q}_{k}$ denote the weighted $\boldsymbol{L}^{2}(\Omega)$-orthogonal projector onto $\overline{\boldsymbol{V}}_{k, 0}$ defined by

$$
\left(\epsilon \boldsymbol{Q}_{1} \boldsymbol{v}, \boldsymbol{\phi}\right)=(\epsilon \boldsymbol{v}, \boldsymbol{\phi}) \quad \text { for all } \boldsymbol{\phi} \in \overline{\boldsymbol{V}}_{1,0}
$$

and

$$
\left(\mu \boldsymbol{Q}_{2} \boldsymbol{v}, \boldsymbol{\phi}\right)=(\mu \boldsymbol{v}, \boldsymbol{\phi}) \quad \text { for all } \boldsymbol{\phi} \in \overline{\boldsymbol{V}}_{2,0}
$$

Then for $\boldsymbol{g}_{k} \in \boldsymbol{L}^{2}(\Omega)$, the functionals given by

$$
\left\langle\boldsymbol{f}_{1}, \boldsymbol{\phi}\right\rangle=\left(\epsilon \boldsymbol{g}_{1}, \boldsymbol{\phi}\right) \quad \text { and } \quad\left\langle\boldsymbol{f}_{2}, \boldsymbol{\phi}\right\rangle=\left(\mu \boldsymbol{g}_{2}, \boldsymbol{\phi}\right) .
$$

satisfy the compatibility condition (2.8) if and only if $\left(\epsilon \boldsymbol{g}_{1}, \boldsymbol{\phi}\right)=0$ for all $\boldsymbol{\phi} \in \overline{\boldsymbol{V}}_{1,0}$ and $\left(\mu \boldsymbol{g}_{2}, \boldsymbol{\phi}\right)=0$ for all $\boldsymbol{\phi} \in \overline{\boldsymbol{V}}_{2,0}$; i.e., $\boldsymbol{g}_{k} \in \boldsymbol{V}_{k, 0}^{\perp}$, the (weighted) $\boldsymbol{L}^{2}(\Omega)$-orthogonal complement of $\overline{\boldsymbol{V}}_{k, 0}$. If $\boldsymbol{g}_{k}$ is in $\overline{\boldsymbol{V}}_{k, 0}^{\perp}$ and $\boldsymbol{f}_{k}$ is defined by (2.10), then $\boldsymbol{x}_{k}$ defined by (2.7) solves (2.5) and (2.6); i.e., $\boldsymbol{h}=\boldsymbol{x}_{1}$ and $\boldsymbol{e}=\boldsymbol{x}_{2}$.

Let $\boldsymbol{S}_{k}: \boldsymbol{L}^{2}(\Omega) \rightarrow \boldsymbol{L}^{2}(\Omega)$ be defined by $\boldsymbol{S}_{k} \boldsymbol{g}_{k}=\boldsymbol{x}_{k}$ where $\boldsymbol{x}_{k}$ solves the problem

$$
b_{1}\left(\boldsymbol{x}_{1},(\boldsymbol{v}, h)\right)=\left(\epsilon\left(\boldsymbol{I}-\boldsymbol{Q}_{1}\right) \boldsymbol{g}_{1}, \boldsymbol{v}\right) \text { for all }(\boldsymbol{v}, h) \in \boldsymbol{Y}_{1}
$$

and

$$
b_{2}\left(\boldsymbol{x}_{k},(\boldsymbol{v}, h)\right)=\left(\mu\left(\boldsymbol{I}-\boldsymbol{Q}_{2}\right) \boldsymbol{g}_{2}, \boldsymbol{v}\right) \text { for all }(\boldsymbol{v}, h) \in \boldsymbol{Y}_{2} .
$$

It is clear from the above discussion that the compatibility conditions are satisfied so that $\boldsymbol{S}_{k}$ is well defined. Moreover, since $\boldsymbol{g}_{k}$ is in $\boldsymbol{L}^{2}(\Omega), \boldsymbol{S}_{1}\left(\boldsymbol{g}_{1}\right)$ is in $\boldsymbol{H}(\mathbf{c u r l}) \cap$ $\boldsymbol{H}_{0}(\operatorname{div} ; \mu)$ and $\boldsymbol{S}_{2}\left(\boldsymbol{g}_{2}\right)$ is in $\boldsymbol{H}_{0}(\mathbf{c u r l}) \cap \boldsymbol{H}$ (div; $\left.\epsilon\right)$ (cf., [12]). Thus, our regularity assumptions imply that $\boldsymbol{S}_{k}\left(\boldsymbol{g}_{k}\right)$ is in $\left(H^{s}(\Omega)\right)^{3}$ for some $s>0$; i.e., $\boldsymbol{S}_{k}$ is a compact mapping of $\boldsymbol{L}^{2}(\Omega)$ into $\boldsymbol{L}^{2}(\Omega)$.

Note that if $\boldsymbol{e} \in \boldsymbol{H}_{0}$ (curl), $\boldsymbol{h} \in \boldsymbol{H}$ (curl), and $\lambda \neq 0$ satisfy (2.1), then they satisfy (2.5) and (2.6) with $\boldsymbol{g}_{1}=\lambda \boldsymbol{e}$ and $\boldsymbol{g}_{2}=-\lambda \boldsymbol{h}$. Moreover, by (2.2) and (2.4), $\boldsymbol{e} \in \boldsymbol{V}_{1,0}^{\perp}$ and $\boldsymbol{h} \in \boldsymbol{V}_{2,0}^{\perp}$, so $\boldsymbol{g}_{k}=\left(\boldsymbol{I}-\boldsymbol{Q}_{k}\right) \boldsymbol{g}_{k}$ and hence

$$
\begin{aligned}
\boldsymbol{h} & =\boldsymbol{S}_{1}\left(\boldsymbol{g}_{1}\right)=\lambda \boldsymbol{S}_{1}(\boldsymbol{e}), \\
\boldsymbol{e} & =\boldsymbol{S}_{2}\left(\boldsymbol{g}_{2}\right)=-\lambda \boldsymbol{S}_{2}(\boldsymbol{h}) .
\end{aligned}
$$

We rewrite this in block matrix form as

$$
\boldsymbol{B}\left(\begin{array}{l}
\boldsymbol{e} \\
\boldsymbol{h}
\end{array}\right) \equiv\left(\begin{array}{cc}
\mathbf{0} & -\boldsymbol{S}_{2} \\
\boldsymbol{S}_{1} & \mathbf{0}
\end{array}\right)\left(\begin{array}{l}
\boldsymbol{e} \\
\boldsymbol{h}
\end{array}\right)=\sigma\left(\begin{array}{l}
\boldsymbol{e} \\
\boldsymbol{h}
\end{array}\right)
$$

where $\sigma=\lambda^{-1}$. We have shown that the eigenpairs $(\boldsymbol{e}, \boldsymbol{h})$ of (2.1) with nonzero $\lambda$ satisfy (2.11). that

Conversely, let $(\boldsymbol{e}, \boldsymbol{h})$ be a solution of (2.11) with nonzero $\sigma$. It follows from [12]

$(2.12) \nabla \times \boldsymbol{S}_{1} \boldsymbol{g}=\epsilon\left(\boldsymbol{I}-\boldsymbol{Q}_{1}\right) \boldsymbol{g} \quad$ and $\quad \nabla \times \boldsymbol{S}_{2} \boldsymbol{g}=\mu\left(\boldsymbol{I}-\boldsymbol{Q}_{2}\right) \boldsymbol{g} \quad$ for all $\boldsymbol{g} \in \boldsymbol{L}^{2}(\Omega)$.

Thus,

$$
\epsilon\left(\boldsymbol{I}-\boldsymbol{Q}_{1}\right) \boldsymbol{e}=\sigma \nabla \times \boldsymbol{h}
$$

and

$$
\mu\left(\boldsymbol{I}-\boldsymbol{Q}_{2}\right) \boldsymbol{h}=-\sigma \nabla \times \boldsymbol{e} .
$$

Moreover, from the definition of $\boldsymbol{S}_{1}$ and $\boldsymbol{S}_{2}$, it is immediate that

$$
\boldsymbol{S}_{1}(\boldsymbol{g}) \in \boldsymbol{V}_{2,0}^{\perp} \quad \text { and } \quad \boldsymbol{S}_{2}(\boldsymbol{g}) \in \boldsymbol{V}_{1,0}^{\perp}
$$


for any $\boldsymbol{g}$. This implies that $\epsilon\left(\boldsymbol{I}-\boldsymbol{Q}_{1}\right) \boldsymbol{e}=\epsilon \boldsymbol{e}$ and $\mu\left(\boldsymbol{I}-\boldsymbol{Q}_{2}\right) \boldsymbol{h}=\mu \boldsymbol{h}$. Combining the above shows that $(\boldsymbol{e}, \boldsymbol{h})$ satisfy (2.1) with $\lambda=1 / \sigma$. We have proved the following theorem.

Theorem 2.1. The eigenvectors corresponding to nonzero eigenvalues for (2.1) and (2.11) are the same, i.e., $\boldsymbol{e} \in \boldsymbol{H}_{0}(\mathbf{c u r l}), \boldsymbol{h} \in \boldsymbol{H}(\mathbf{c u r l})$, and $\lambda \neq 0$ satisfy (2.1) if and only if they satisfy (2.11) with $\sigma=1 / \lambda$.

Clearly, $\boldsymbol{B}$ is a compact operator mapping $\left(\boldsymbol{L}^{2}(\Omega)\right)^{2}$ into $\left(\boldsymbol{L}^{2}(\Omega)\right)^{2}$. Moreover, if we consider $\boldsymbol{B}$ as an operator on $\boldsymbol{L}_{\epsilon}^{2}(\Omega) \times \boldsymbol{L}_{\mu}^{2}(\Omega)$, with the standard inner product

$$
\left(\left(\begin{array}{l}
\boldsymbol{e} \\
\boldsymbol{h}
\end{array}\right),\left(\begin{array}{c}
\tilde{\boldsymbol{e}} \\
\tilde{\boldsymbol{h}}
\end{array}\right)\right)=(\epsilon \boldsymbol{e}, \tilde{\boldsymbol{e}})+(\mu \boldsymbol{h}, \tilde{\boldsymbol{h}}),
$$

then $\boldsymbol{B}$ is skew-Hermitian. Indeed,

$$
\left(\boldsymbol{B}\left(\begin{array}{l}
\boldsymbol{e} \\
\boldsymbol{h}
\end{array}\right),\left(\begin{array}{c}
\tilde{\boldsymbol{e}} \\
\tilde{\boldsymbol{h}}
\end{array}\right)\right)=-\left(\boldsymbol{S}_{2} \boldsymbol{h}, \epsilon \tilde{\boldsymbol{e}}\right)+\left(\boldsymbol{S}_{1} \boldsymbol{e}, \mu \tilde{\boldsymbol{h}}\right) .
$$

Using (2.12) and (2.13) gives

$$
\begin{aligned}
\left(\boldsymbol{S}_{2} \boldsymbol{h}, \epsilon \tilde{\boldsymbol{e}}\right) & =\left(\boldsymbol{S}_{2} \boldsymbol{h}, \epsilon\left(\boldsymbol{I}-\boldsymbol{Q}_{1}\right) \tilde{\boldsymbol{e}}\right)=\left(\boldsymbol{S}_{2} \boldsymbol{h}, \nabla \times \boldsymbol{S}_{1} \tilde{\boldsymbol{e}}\right) \\
& =\left(\nabla \times \boldsymbol{S}_{2} \boldsymbol{h}, \boldsymbol{S}_{1} \tilde{\boldsymbol{e}}\right)=\left(\mu\left(\boldsymbol{I}-\boldsymbol{Q}_{2}\right) \boldsymbol{h}, \boldsymbol{S}_{1} \tilde{\boldsymbol{e}}\right)=\left(\mu \boldsymbol{h}, \boldsymbol{S}_{1} \tilde{\boldsymbol{e}}\right)
\end{aligned}
$$

from which it follows that $\boldsymbol{B}$ is skew-Hermitian. Note that the above identity is just $\boldsymbol{S}_{1}^{*}=\boldsymbol{S}_{2}$, where $\boldsymbol{S}_{1}$ is considered as an operator from $\boldsymbol{L}_{\epsilon}^{2}(\Omega)$ to $\boldsymbol{L}_{\mu}^{2}(\Omega)$. When $\boldsymbol{S}_{1}$ is considered as an operator on $\boldsymbol{L}^{2}(\Omega)$, we will denote its adjoint by $\boldsymbol{S}_{1}^{\boldsymbol{t}}$.

The eigenvectors and eigenvalues of $\boldsymbol{B}$ are related to the compact positive semidefinite operator

$$
-\boldsymbol{B}^{2}=\left(\begin{array}{cc}
\boldsymbol{S}_{2} \boldsymbol{S}_{1} & \mathbf{0} \\
\mathbf{0} & \boldsymbol{S}_{1} \boldsymbol{S}_{2}
\end{array}\right) .
$$

This operator is Hermitian relative to the inner product on $\boldsymbol{L}_{\epsilon}^{2}(\Omega) \times \boldsymbol{L}_{\mu}^{2}(\Omega)$. The nonzero eigenvalues and the corresponding eigenvectors for $\boldsymbol{B}$ can be recovered also from those of either diagonal block above. For example, $\boldsymbol{S}_{2} \boldsymbol{S}_{1}: \boldsymbol{L}^{2}(\Omega) \rightarrow \boldsymbol{L}^{2}(\Omega)$ is Hermitian relative to the inner product $(\epsilon \cdot, \cdot)$ and if the real function $\phi$ satisfies

$$
\boldsymbol{S}_{2} \boldsymbol{S}_{1} \boldsymbol{\phi}=\tau^{2} \boldsymbol{\phi}
$$

then

$$
\left(\begin{array}{c}
\phi \\
\frac{i}{\tau} \boldsymbol{S}_{1} \phi
\end{array}\right) \text { and }\left(\begin{array}{c}
\phi \\
\frac{-i}{\tau} \boldsymbol{S}_{1} \phi
\end{array}\right)
$$

are eigenvectors for $\boldsymbol{B}$ with eigenvalues $-i \tau$ and $i \tau$, respectively. We get all nonzero eigenvalues and their corresponding eigenvectors this way. Thus, the original eigenvalue problem (2.1) can be reduced to the real eigenvalue problem (2.15). The eigenvectors and nonzero eigenvalues of $\boldsymbol{B}$ can be recovered from those of the lower right diagonal block of $-\boldsymbol{B}^{2}$ in an analogous fashion.

Remark 2.1. The form (with $\omega \neq 0$ )

$$
b^{\omega}\left(\boldsymbol{h}, \boldsymbol{e}, \boldsymbol{v}_{1}, \boldsymbol{v}_{2}, h_{1}, h_{2}\right)=b_{1}\left(\boldsymbol{h} ; \boldsymbol{v}_{1}, h_{1}\right)-\omega\left(\epsilon \boldsymbol{e}, \boldsymbol{v}_{1}\right)+b_{2}\left(\boldsymbol{e} ; \boldsymbol{v}_{2}, h_{2}\right)+\omega\left(\mu \boldsymbol{h}, \boldsymbol{v}_{2}\right)
$$

(for $\boldsymbol{h}, \boldsymbol{e} \in \boldsymbol{L}^{2}(\Omega), \boldsymbol{v}_{k} \in \boldsymbol{V}_{k}, h_{k} \in H_{k}, k=1,2$ ) arises in an analysis of the time harmonic Maxwell problem given in [9]. Suppose that nonzero $\boldsymbol{h}, \boldsymbol{e} \in \boldsymbol{L}^{2}(\Omega)$ satisfy

$$
b^{\omega}\left(\boldsymbol{h}, \boldsymbol{e}, \boldsymbol{v}_{1}, \boldsymbol{v}_{2}, h_{1}, h_{2}\right)=0
$$


for all $\boldsymbol{v}_{k} \in \boldsymbol{V}_{k}, h_{k} \in H_{k}$. Then $\boldsymbol{x}_{1}=\boldsymbol{h}$ and $\boldsymbol{x}_{2}=\boldsymbol{e}$ satisfy (2.5) and (2.6) with $\boldsymbol{g}_{1}=\omega \boldsymbol{e}$ and $\boldsymbol{g}_{2}=-\omega \boldsymbol{h}$. Arguments given above imply that $\boldsymbol{e} \in \boldsymbol{H}_{0}$ (curl), $\boldsymbol{h} \in \boldsymbol{H}$ (curl) and satisfy (2.1) with $\lambda$ replaced by $\omega$; i.e., $\omega^{-1}$ is an eigenvalue of $\boldsymbol{B}$. It follows that if $\omega^{-1}$ is not an eigenvalue of $\boldsymbol{B}$, then there are no nontrivial solutions of (2.16).

\section{Approximation of the operators $\boldsymbol{S}_{k}, k=1,2$}

To define approximations to $\boldsymbol{S}_{k}$, we start with a finite-element approximation given in 12 for problem (2.7). There were two techniques discussed in (2.7) and we shall only consider the first involving stable pairs of approximation spaces. The second technique in (2.7) involving stabilization by form modification could also be applied.

In [12, it is shown how problem (2.7) can be discretized using pairs of approximation subspaces, $\boldsymbol{X}_{h} \subset \boldsymbol{L}^{2}(\Omega)$ and $\boldsymbol{Y}_{h, k} \subset \boldsymbol{Y}_{k}$. These spaces are constructed so that $\boldsymbol{X}_{h}$ has approximation properties and the pair satisfies the discrete inf-sup condition

$$
\left\|\boldsymbol{x}_{k}\right\|_{\boldsymbol{L}^{2}(\Omega)} \leq C \sup _{(\boldsymbol{v}, h) \in \boldsymbol{Y}_{k}} \frac{b_{k}\left(\boldsymbol{x}_{k},(\boldsymbol{v}, h)\right)}{\|(\boldsymbol{v}, h)\|_{\boldsymbol{Y}_{h, k}}} \quad \text { for all } \boldsymbol{x}_{k} \in \boldsymbol{X}_{h} .
$$

The simplest discretization involved locally quasi-uniform partitioning of the computational domain into tetrahedra and setting $\boldsymbol{X}_{h}$ to be the space of piecewise constant vector fields with respect to the mesh (cf., [12]). The companion spaces $\boldsymbol{Y}_{h, k}$ consist of continuous piecewise linear functions (satisfying the appropriate boundary conditions) enriched with face bubble functions. For more detail, see 12. The addition of the bubble functions gives rise to (3.1) much in the same way as the addition of bubble functions are used to stabilize Stokes approximations [20].

One can then consider the discrete problem: Find $\boldsymbol{x}_{h, k} \in \boldsymbol{X}_{h}$ satisfying

$$
b_{k}\left(\boldsymbol{x}_{h, k}, \boldsymbol{y}\right)=\left\langle\boldsymbol{f}_{k}, \boldsymbol{y}\right\rangle \quad \text { for all } \boldsymbol{y} \in \boldsymbol{Y}_{h, k} .
$$

The above equation, however, has a solution only if the data $\boldsymbol{f}_{k}$ satisfy appropriate discrete compatibility conditions. Such conditions are difficult to deal with in practice, and so instead we use an approach that is related to least-squares in a dual norm.

To define this approximation, we first set $\boldsymbol{T}_{h, k}: \boldsymbol{Y}_{h, k}^{*} \rightarrow \boldsymbol{Y}_{h, k}$ by

$$
\left(\boldsymbol{T}_{h, k} l, \boldsymbol{y}\right)_{1}=\langle l, \boldsymbol{y}\rangle \quad \text { for all } \boldsymbol{y} \in \boldsymbol{Y}_{h, k} .
$$

Here $(\cdot, \cdot)_{1}$ denotes the componentwise $H^{1}$-inner product in $\boldsymbol{Y}_{h, k}$. The approximation $\boldsymbol{x}_{h, k}$ is then defined to be the unique function in $\boldsymbol{X}_{h}$ satisfying

$$
\boldsymbol{A}_{h, k}\left(\boldsymbol{x}_{h, k}, \boldsymbol{x}\right) \equiv\left\langle\boldsymbol{B}_{h, k} \boldsymbol{x}_{h, k}, \boldsymbol{T}_{h, k} \boldsymbol{B}_{h, k} \boldsymbol{x}\right\rangle=\left\langle\boldsymbol{f}_{k}, \boldsymbol{T}_{h, k} \boldsymbol{B}_{h, k} \boldsymbol{x}\right\rangle, \quad \text { for all } \boldsymbol{x} \in \boldsymbol{X}_{h} .
$$

Here $\boldsymbol{B}_{h, k}$ is the map of $\boldsymbol{X}_{h}$ into $\boldsymbol{Y}_{h, k}^{*}$ defined by

$$
\left\langle\boldsymbol{B}_{h, k} \boldsymbol{x}, \boldsymbol{y}\right\rangle=b_{k}(\boldsymbol{x}, \boldsymbol{y}) \quad \text { for all } \boldsymbol{y} \in \boldsymbol{Y}_{h, k} .
$$

The existence, uniqueness, and convergence properties of the above approximation are provided in [12. There it is shown that if $\boldsymbol{f}_{k}$ satisfies the compatibility condition (2.8) and the solution $\boldsymbol{x}_{k}$ of (2.7) is in $\left(H^{s}(\Omega)\right)^{3}$ for some $s \in[0,1]$, then

$$
\left\|\boldsymbol{x}_{k}-\boldsymbol{x}_{h, k}\right\| \leq C h^{s}\left\|\boldsymbol{x}_{k}\right\|_{s}
$$


For later use, we let $\boldsymbol{R}_{h, k}$ denote the operator on $\boldsymbol{L}^{2}(\Omega)$ defined by $\boldsymbol{R}_{h, k} \boldsymbol{w}=\boldsymbol{x}_{h, k}$ where $\boldsymbol{x}_{h, k}$ solves (3.2) with data

$$
\left\langle\boldsymbol{f}_{1},(\boldsymbol{v}, h)\right\rangle=(\epsilon \boldsymbol{w}, \boldsymbol{v})
$$

and

$$
\left\langle\boldsymbol{f}_{2},(\boldsymbol{v}, h)\right\rangle=(\mu \boldsymbol{w}, \boldsymbol{v}) .
$$

Evaluation of the discrete operator $\boldsymbol{T}_{h, k}$ involves solving finite-element approximations to second-order problems with various boundary conditions using the subspaces enriched by bubble functions. It is shown in 12 that these problems can be replaced by efficient preconditioners. The computation of the solution $\boldsymbol{x}_{h, k}$ can then be obtained by an efficient, well-conditioned (rapidly convergent), preconditioned, conjugate gradient iteration.

To define our approximation for $\boldsymbol{S}_{k}$, we set $\boldsymbol{f}_{k}$ in (3.2) by

$$
\left\langle\boldsymbol{f}_{1},(\boldsymbol{v}, h)\right\rangle=\left(\epsilon\left(\boldsymbol{I}-\boldsymbol{Q}_{h, 1}\right) \boldsymbol{g}_{1}, \boldsymbol{v}\right) \quad \text { and }\left\langle\boldsymbol{f}_{2},(\boldsymbol{v}, h)\right\rangle=\left(\mu\left(\boldsymbol{I}-\boldsymbol{Q}_{h, 1}\right) \boldsymbol{g}_{2}, \boldsymbol{v}\right),
$$

and define $\boldsymbol{S}_{h, k} \boldsymbol{g}_{k}=\boldsymbol{x}_{h, k}$. The operators $\boldsymbol{Q}_{h, k}, k=1,2$, are defined in terms of the approximation subspace for $\overline{\boldsymbol{V}}_{k, 0}$. For example, if $H_{h, k}$ is the approximation subspace associated with $\boldsymbol{Y}_{h, k}$, then we define $\boldsymbol{Q}_{h, 1} \boldsymbol{v}=\nabla \phi$ where $\phi \in H_{h, 2}$ satisfies

$$
(\epsilon \nabla \phi, \nabla \theta)=(\epsilon \boldsymbol{v}, \nabla \theta) \quad \text { for all } \theta \in H_{h, 2} .
$$

Similarly, we define $\boldsymbol{Q}_{h, 2} \boldsymbol{v}=\nabla \phi$ where $\phi \in H_{h, 1}$ satisfies

$$
(\mu \nabla \phi, \nabla \theta)=(\mu \boldsymbol{v}, \nabla \theta) \text { for all } \theta \in H_{h, 1} .
$$

Actually, as will become clear later, the bubble functions are not needed for $\boldsymbol{Q}_{h, k}$. For example, for the case when $\boldsymbol{X}_{h}$ is piecewise constant, it suffices to use the subspaces of piecewise linear functions with appropriate boundary conditions in the definition of $\boldsymbol{Q}_{h, k}$. However, the bubble functions are still required in the spaces $\boldsymbol{Y}_{h, k}$.

To analyze the approximation properties of the above operators, we shall need regularity results for second-order problems with piecewise smooth coefficients. For example, we consider the solution $u \in H_{0}^{1}(\Omega)$ satisfying

$$
(\epsilon \nabla u, \nabla \phi)=\langle f, \phi\rangle \quad \text { for all } \phi \in H_{0}^{1}(\Omega) .
$$

We assume that the solution is in $H^{1+s}(\Omega)$ when $f$ coincides with a function in $L^{2}(\Omega)$; i.e.,

$$
\|u\|_{1+s} \leq C\|f\| .
$$

Estimates of this sort were given in [17] for piecewise smooth $\epsilon$.

We also assume similar regularity for the Neumann problem involving $\mu$. For $f \in\left(H^{1}(\Omega)\right)^{*}$ with $\langle f, 1\rangle=0$, let $u \in H^{1}(\Omega)$ have zero mean value and satisfy

$$
(\mu \nabla u, \nabla \phi)=\langle f, \phi\rangle \quad \text { for all } \phi \in H^{1}(\Omega) .
$$

We assume that the solution is in $H^{1+s}(\Omega)$ when $f$ coincides with a function in $L^{2}(\Omega)$; i.e., (3.7) holds for $u$ solving (3.8).

Remark 3.1. We assume that multiplication by $\epsilon$ and $\mu$ are bounded operators on $H^{\gamma}(\Omega)$. This holds for $0<\gamma<1 / 2$ when the coefficients are piecewise smooth with respect to polygonal subdomains $\left\{\Omega_{j}\right\}$. Indeed, for $0<\gamma<1 / 2$ and Lipschitz continuous domains $D, H^{\gamma}(D)=H_{0}^{\gamma}(D)$ from which it follows by interpolation between $\sum H_{0}^{1}\left(\Omega_{j}\right)$ and $L^{2}(\Omega)$ that $H^{\gamma}(\Omega)$ is isomorphic to $\sum_{j} H^{\gamma}\left(\Omega_{j}\right)$. Since $\epsilon$ is 
piecewise smooth, multiplication by $\epsilon$ is a bounded operator on $\sum_{j} H^{\gamma}\left(\Omega_{j}\right)$. For smooth coefficients, we can take $\gamma=1$.

We then have the following theorem.

Theorem 3.1. Let $\gamma$ be such that multiplication by $\epsilon$ and $\mu$ are bounded operators on $H^{\gamma}(\Omega)$ (see Remark 3.1). There is a positive constant $C=C(\gamma)$ independent of $h$ such that for $k=1,2$,

$$
\left\|\boldsymbol{S}_{k}-\boldsymbol{S}_{h, k}\right\| \leq C h^{s \gamma}
$$

Here $\|\cdot\|$ denotes the operator norm on $\boldsymbol{L}^{2}(\Omega)$.

Proof. We consider $k=1$. The case of $k=2$ is similar. We have that for $\boldsymbol{g}_{1} \in$ $\boldsymbol{L}^{2}(\Omega)$,

$$
\boldsymbol{S}_{1} \boldsymbol{g}_{1}-\boldsymbol{S}_{h, 1} \boldsymbol{g}_{1}=\boldsymbol{x}_{1}-\boldsymbol{x}_{h, 1}+\boldsymbol{R}_{h, 1}\left(\boldsymbol{Q}_{h, 1}-\boldsymbol{Q}_{1}\right) \boldsymbol{g}_{1}
$$

where $\boldsymbol{x}_{1}$ and $\boldsymbol{x}_{h, 1}$ solve (2.7) and (3.2), respectively, with

$$
\left\langle\boldsymbol{f}_{1},(\boldsymbol{v}, h)\right\rangle=\left(\epsilon\left(\boldsymbol{I}-\boldsymbol{Q}_{1}\right) \boldsymbol{g}_{1}, \boldsymbol{v}\right) .
$$

By (3.3) and our regularity assumptions on $\boldsymbol{S}_{1}$,

$$
\left\|\boldsymbol{x}_{1}-\boldsymbol{x}_{h, 1}\right\| \leq C h^{s}\left\|\boldsymbol{x}_{1}\right\|_{\left(H^{s}(\Omega)\right)^{3}} \leq C h^{s}\left\|\boldsymbol{g}_{1}\right\| .
$$

Let $\boldsymbol{w}=\left(\boldsymbol{Q}_{h, 1}-\boldsymbol{Q}_{1}\right) \boldsymbol{g}_{1}$. To complete the proof, we need only show that

$$
\left\|\boldsymbol{R}_{h, 1} \boldsymbol{w}\right\| \leq C h^{s \gamma}\left\|\boldsymbol{g}_{1}\right\| .
$$

It is shown in 12, that the form $\boldsymbol{A}_{h, 1}(\boldsymbol{x}, \boldsymbol{x})$ is equivalent to $\|\boldsymbol{x}\|^{2}$ for $\boldsymbol{x} \in \boldsymbol{X}_{h}$ uniformly in $h$. Thus,

$$
\begin{aligned}
\left\|\boldsymbol{R}_{h, 1} \boldsymbol{w}\right\|^{2} & \leq C \boldsymbol{A}_{h, 1}\left(\boldsymbol{R}_{h, 1} \boldsymbol{w}, \boldsymbol{R}_{h, 1} \boldsymbol{w}\right) \\
& =\left(\epsilon \boldsymbol{w}, \boldsymbol{T}_{h, 1} \boldsymbol{B}_{h, 1} \boldsymbol{R}_{h, 1} \boldsymbol{w}\right) \leq\|\boldsymbol{w}\|_{-\gamma}\left\|\epsilon \boldsymbol{T}_{h, 1} \boldsymbol{B}_{h, 1} \boldsymbol{R}_{h, 1} \boldsymbol{w}\right\|_{\gamma} \\
& \leq C\|\boldsymbol{w}\|_{-\gamma}\left\|\boldsymbol{T}_{h, 1} \boldsymbol{B}_{h, 1} \boldsymbol{R}_{h, 1} \boldsymbol{w}\right\|_{\gamma}
\end{aligned}
$$

Note that

$$
\begin{aligned}
\left\|\boldsymbol{T}_{h, 1} \boldsymbol{B}_{h, 1} \boldsymbol{R}_{h, 1} \boldsymbol{w}\right\|_{\gamma} & \leq\left\|\boldsymbol{T}_{h, 1} \boldsymbol{B}_{h, 1} \boldsymbol{R}_{h, 1} \boldsymbol{w}\right\|_{1} \\
& \leq C \boldsymbol{A}_{h, 1}\left(\boldsymbol{R}_{h, 1} \boldsymbol{w}, \boldsymbol{R}_{h, 1} \boldsymbol{w}\right)^{\frac{1}{2}} \leq C\left\|\boldsymbol{R}_{h, 1} \boldsymbol{w}\right\|
\end{aligned}
$$

so we need only bound $\|\boldsymbol{w}\|_{-\gamma}$. The middle inequality follows from the definition of $\boldsymbol{T}_{h, k}$ and (3.2).

Now $\boldsymbol{Q}_{1} \boldsymbol{g}_{1}=\nabla u$, where $u \in H_{0}^{1}(\Omega)$ satisfies (3.6) with $\langle f, \theta\rangle=\left(\epsilon \boldsymbol{g}_{1}, \nabla \theta\right)$. In addition $\boldsymbol{Q}_{h, 1} \boldsymbol{g}_{1}=\nabla u_{h}$, where $u_{h}$ is the elliptic projection of $u$ into $H_{h, 2}$; i.e., $\boldsymbol{w}=\nabla\left(u-u_{h}\right)$. Now

$$
\left\|\nabla\left(u-u_{h}\right)\right\| \leq C\left\|\boldsymbol{g}_{1}\right\| .
$$

Furthermore, by finite element duality and (3.7),

$$
\left\|\nabla\left(u-u_{h}\right)\right\|_{-1} \leq\left\|u-u_{h}\right\| \leq C h^{s}\|u\|_{1} \leq C h^{s}\left\|\boldsymbol{g}_{1}\right\| .
$$

By interpolation

$$
\|\boldsymbol{w}\|_{-\gamma}=\left\|\nabla\left(u-u_{h}\right)\right\|_{-\gamma} \leq C h^{s \gamma}\left\|\boldsymbol{g}_{1}\right\| .
$$

The inequality (3.9) follows combining the above estimates. This completes the proof of the theorem. 
Remark 3.2. If we assume stronger shift theorems, e.g.,

$$
\|u\|_{1+s} \leq C\|f\|_{-1+s}
$$

for all $f \in H^{-1+s}(\Omega)$ (for $0<s<s_{0}$ ), then by finite element duality

$$
\left\|\nabla\left(u-u_{h}\right)\right\|_{-s} \leq\left\|u-u_{h}\right\|_{1-s} \leq C h^{s}\|u\|_{1} .
$$

Thus, the theorem becomes

$$
\left\|\boldsymbol{S}_{k}-\boldsymbol{S}_{k, h}\right\| \leq C h^{\min (\gamma, s)} .
$$

\section{The eigenvalue/eigenvector Discretization}

In this section we use the approximations and results of the previous section to define and analyze an approximation to the eigenvalue/eigenvector problem (2.1). As previously observed, this reduces to approximating the eigenvalues and eigenvectors for either of the symmetric semi-definite operators $\boldsymbol{S}_{2} \boldsymbol{S}_{1}$ or $\boldsymbol{S}_{1} \boldsymbol{S}_{2}$. We could directly use the discrete operators $\boldsymbol{S}_{h, k}, k=1,2$. This will be avoided for two reasons. One would have to code both $\boldsymbol{S}_{h, 1}$ and $\boldsymbol{S}_{h, 2}$. In addition, even though the product of the continuous operators is symmetric, the product of their discrete counterparts is not likely to be symmetric.

We circumvent the above mentioned problems by implementing only one of the discrete operators, e.g., $\boldsymbol{S}_{h, 1}$. Then, instead of implementing $\boldsymbol{S}_{h, 2}$, we implement the adjoint $\boldsymbol{S}_{h, 1}^{*}$ of $\boldsymbol{S}_{h, 1}$, considered as an operator of $\boldsymbol{L}_{\epsilon}^{2}(\Omega)$ into $\boldsymbol{L}_{\mu}^{2}(\Omega)$. The implementation of $\boldsymbol{S}_{h, 1}^{*}=\epsilon^{-1} \boldsymbol{S}_{h, 1}^{t} \mu$ is relatively straightforward given the implementation of $\boldsymbol{S}_{h, 1}$. Indeed, $\boldsymbol{S}_{h, 1}$ is implemented as a sequence of matrix operations, and the implementation of $\boldsymbol{S}_{h, 1}^{t}$ just reduces to transposing the matrix operations and running them in reverse order. Note that $\boldsymbol{S}_{h, 1}^{*} \boldsymbol{S}_{h, 1}$ is symmetric by definition.

The symmetry of the approximation is an important property. This is because realistic computations for three-dimensional electromagnetic devices necessarily involve minimal problem sizes on the order of $10^{6}$ unknowns. The eigenvalues and eigenvectors of such systems cannot be computed by direct methods. It is often of interest to compute a block of the smallest nonzero eigenvalues and eigenvectors of (2.1) 23, 38. This means that we are required to iteratively compute the largest eigenvalues and their corresponding eigenvectors for the problem $\boldsymbol{S}_{h, 1}^{*} \boldsymbol{S}_{h, 1} \boldsymbol{x}=\tau^{2} \boldsymbol{x}$. The problem of iteratively computing the largest eigenvalues of a symmetric positive semi-definite problem has been well studied; see, for example, 8, 26, 18, 28]. Even block versions of the power method work, although not as well as other iterative strategies. A survey of iterative methods for eigenvalue problems can be found in [27].

By Theorem $3.1 \boldsymbol{S}_{h, 1}$ converges to $\boldsymbol{S}_{1}$ in norm. It immediately follows that $\boldsymbol{S}_{h, 1}^{*}$ converges to $\boldsymbol{S}_{1}^{*}=\boldsymbol{S}_{2}$. It follows from the identity

$$
\boldsymbol{S}_{h, 1}^{*} \boldsymbol{S}_{h, 1}-\boldsymbol{S}_{2} \boldsymbol{S}_{1}=\left(\boldsymbol{S}_{h, 1}^{*}-\boldsymbol{S}_{2}\right) \boldsymbol{S}_{h, 1}+\boldsymbol{S}_{2}\left(\boldsymbol{S}_{h, 1}-\boldsymbol{S}_{1}\right)
$$

that $\boldsymbol{S}_{h, 1}^{*} \boldsymbol{S}_{h, 1}$ converges to $\boldsymbol{S}_{2} \boldsymbol{S}_{1}$ in norm. By standard perturbation theory [25], one can conclude that if $\tau^{2}>0$ is an eigenvalue of $\boldsymbol{S}_{2} \boldsymbol{S}_{1}$ of multiplicity $k$ and $\nu>0$ is given such that there are no other eigenvalues in the interval $\delta=\left(\tau^{2}-\nu, \tau^{2}+\nu\right)$, then for $h$ small enough there will be exactly $k$ discrete eigenvalues $\left\{\tau_{i}^{2}(h)\right\}_{i=1}^{k}$ (counted up to multiplicity) in $\delta$. Thus, there will not be any spurious discrete eigenvalues. 
Alternatively, we can use $\boldsymbol{S}_{h, 1} \boldsymbol{S}_{h, 1}^{*}$ to approximate $\boldsymbol{S}_{1} \boldsymbol{S}_{2}, \boldsymbol{S}_{h, 2} \boldsymbol{S}_{h, 2}^{*}$ to approximate $\boldsymbol{S}_{2} \boldsymbol{S}_{1}$, or $\boldsymbol{S}_{h, 2}^{*} \boldsymbol{S}_{h, 2}$ to approximate $\boldsymbol{S}_{1} \boldsymbol{S}_{2}$. The analogous results for eigenvalue/eigenvector convergence follow for these operators as well.

Next we discuss the eigenvector and eigenvalue convergence rate. Using the general results for spectral approximation of compact operators (see, e.g., [11]), we get that there is a constant $C=C(\tau)>0$ such that if $V$ is the eigenspace corresponding to $\tau^{2}$ and $V_{h}$ is the eigenspace corresponding to the eigenvalues of $\boldsymbol{S}_{h, 1}^{*} \boldsymbol{S}_{h, 1}$ in $\delta$, then for small enough $h$

$$
\hat{\delta}\left(V, V_{h}\right) \equiv \sup _{v \in V,\|v\|=1} \operatorname{dist}\left(v, V_{h}\right) \leq C\left\|\boldsymbol{S}_{2} \boldsymbol{S}_{1}-\boldsymbol{S}_{h, 1}^{*} \boldsymbol{S}_{h, 1}\right\| .
$$

The quantity $\hat{\delta}\left(V, V_{h}\right)$ is called the gap between $V$ and $V_{h}$. It is a measure for closeness of subspaces, which in this case is related to the angle between them. Further detail and results concerning $\hat{\delta}$ can be found in 25]. Related estimates, demonstrating that each orthonormal basis of $V$ can be approximated by an orthonormal basis of $V_{h}$, with the same rate, are given in [11, pp. 532-533].

Combining (4.1) with Theorem 3.1 we obtain the following convergence result for the eigenvectors.

Theorem 4.1. Let $\omega>0$ be fixed, such that $\lambda=i \omega$ is an eigenvalue of (2.1). Let $\tau=\omega^{-1}$, and $V, V_{h}$ are the eigenspaces defined above. Then, for small enough $h$, there is a positive constant $C=C(\omega)$ independent of $h$ such that

$$
\hat{\delta}\left(V, V_{h}\right) \leq C h^{s \gamma} .
$$

Regarding the eigenvalues, the general theory states (cf. 25]) that there exists a constant $C=C(\tau)>0$, such that if $h$ is small enough,

$$
\left|\tau^{2}-\tau_{i}^{2}(h)\right| \leq C\left\|\boldsymbol{S}_{2} \boldsymbol{S}_{1}-\boldsymbol{S}_{h, 1}^{*} \boldsymbol{S}_{h, 1}\right\|
$$

for all $i=1, \ldots, k$. Thus, in general, we get the following convergence result for the eigenvalues.

Theorem 4.2. Let $\omega>0$ be fixed, such that $\lambda=i \omega$ is an eigenvalue of (2.1). Let $\tau=\omega^{-1}$, and $\left\{\tau_{j}^{2}(h)\right\}_{j=1}^{k}$ are the eigenvalues defined above. Then, for small enough $h$, there is a positive constant $C=C(\omega)$ independent of $h$ such that for all $j=1, \ldots, k$,

$$
\left|\tau^{2}-\tau_{j}^{2}(h)\right| \leq C h^{s \gamma} .
$$

Our numerical results, however, indicate that sometimes the rate of convergence of the eigenvalues is significantly better than the rate of convergence of the eigenvectors. Below we outline a proof of this fact in the case of smooth eigenvectors.

For the remainder of this section we assume that $\Omega$ is a convex polyhedron, $\epsilon=\mu=1, \boldsymbol{T}_{h, k}$ corresponds to a direct solve (not a preconditioner), and the eigenvectors are such that $\boldsymbol{e} \cdot \boldsymbol{n} \in H^{3 / 2}(F)$ on each face $F$ of $\partial \Omega$. This is the case, for example, if the domain is the unit cube. By Theorem 3.1 we have $\left\|\boldsymbol{S}_{k}-\boldsymbol{S}_{h, k}\right\| \leq C h$ for $k=1,2$.

Fix an eigenvector $\boldsymbol{e}$ of $\boldsymbol{S}_{2} \boldsymbol{S}_{1}$ corresponding to an eigenvalue $\tau^{2}$ and let $0<\varepsilon<\frac{1}{2}$. We will prove that the approximation of $\tau^{2}$ converges at a rate of at least $h^{2-\varepsilon}$. 
Consider the biharmonic problem

$$
\begin{array}{rlrl}
\Delta^{2} \psi & =0 & & \text { in } \Omega, \\
\psi=0 & & \text { on } \partial \Omega, \\
\frac{\partial \psi}{\partial n}=\theta & & \text { on } \partial \Omega,
\end{array}
$$

with data $\theta=\boldsymbol{e} \cdot \boldsymbol{n}$. By our assumptions, $\theta \in H^{3 / 2}(F)$ and $\theta=0$ on $\partial F$ on every face $F$ of $\partial \Omega$. By examination of the proof of the regularity result from [21, one can show that this implies $\psi \in H^{3-\varepsilon}(\Omega)$.

Set $\boldsymbol{w}=\tau^{-2} \boldsymbol{e}+\nabla \Delta \psi$ and consider the div-curl system

$$
\begin{aligned}
\nabla \times \boldsymbol{v} & =\boldsymbol{w} \quad \text { in } \Omega, \\
\nabla \cdot \boldsymbol{v} & =0 \quad \text { in } \Omega, \\
\boldsymbol{v} \cdot \boldsymbol{n} & =0 \quad \text { on } \partial \Omega .
\end{aligned}
$$

By construction, $\boldsymbol{w}$ is in $\boldsymbol{H}^{-\varepsilon}(\Omega)$, the dual space of $H_{0}^{\varepsilon}(\Omega)^{3}$, and satisfies the compatability conditions, so the above problem is well posed. Moreover, we show in the appendix that the solution is in $\boldsymbol{H}^{1-\varepsilon}(\Omega)$ and there exist $C>0$ such that $\|\boldsymbol{v}\|_{1-\varepsilon} \leq C\|\boldsymbol{w}\|_{\varepsilon}$.

Define $\boldsymbol{T}_{1}: \boldsymbol{H}^{-1}(\Omega) \mapsto \boldsymbol{H}_{0}^{1}(\Omega)$ by

$$
\left(\nabla \boldsymbol{T}_{1} \ell, \nabla \boldsymbol{z}\right)=\langle\ell, \boldsymbol{z}\rangle \quad \text { for all } \boldsymbol{z} \in \boldsymbol{H}_{0}^{1}(\Omega) .
$$

We claim that

$$
\nabla \times \boldsymbol{T}_{1} \nabla \times \boldsymbol{v}=\nabla \times \boldsymbol{e} .
$$

Indeed, $\boldsymbol{e}-\nabla \psi \in \boldsymbol{H}_{0}^{1}(\Omega)$ by (4.3), and therefore

$$
\left.\boldsymbol{e}-\nabla \psi=\boldsymbol{T}_{1}(-\Delta(\boldsymbol{e}-\nabla \psi))=\boldsymbol{T}_{1}\left(\tau^{-2} \boldsymbol{e}+\nabla \Delta \psi\right)\right) .
$$

The result follows by applying the curl operator to both sides.

Let $\tau_{h}^{2}$ and $\boldsymbol{e}_{h}$ be the eigenvalue and eigenvector approximations to $\tau^{2}$ and $\boldsymbol{e}$, respectively. Set $\boldsymbol{u}=\left(\boldsymbol{e}, \tau^{-1} \boldsymbol{S}_{1} \boldsymbol{e}\right)^{t}$ and $\boldsymbol{u}_{h}=\left(\boldsymbol{e}_{h}, \tau_{h}^{-1} \boldsymbol{S}_{h, 1} \boldsymbol{e}_{h}\right)^{t}$. We assume that $\boldsymbol{u}$ and $\boldsymbol{u}_{h}$ are scaled so that $\|\boldsymbol{u}\|=\left\|\boldsymbol{u}_{h}\right\|=1$, where $\|\cdot\|$ denotes the square root of the sum of the squares of the $\boldsymbol{L}^{2}(\Omega)$-norms on the two components. We then have $\tilde{\boldsymbol{B}} \boldsymbol{u}=\tau \boldsymbol{u}$ and $\tilde{\boldsymbol{B}}_{h} \boldsymbol{u}_{h}=\tau_{h} \boldsymbol{u}_{h}$, where

$$
\tilde{\boldsymbol{B}} \equiv\left(\begin{array}{cc}
\mathbf{0} & \boldsymbol{S}_{2} \\
\boldsymbol{S}_{1} & \mathbf{0}
\end{array}\right) \quad \text { and } \quad \tilde{\boldsymbol{B}}_{h} \equiv\left(\begin{array}{cc}
\mathbf{0} & \boldsymbol{S}_{h, 1}^{*} \\
\boldsymbol{S}_{h, 1} & \mathbf{0}
\end{array}\right)
$$

Simple algebraic manipulations show that

$$
\begin{aligned}
\tau-\tau_{h}= & \left((\tau \boldsymbol{I}-\tilde{\boldsymbol{B}})\left(\boldsymbol{u}-\boldsymbol{u}_{h}\right), \boldsymbol{u}-\boldsymbol{u}_{h}\right) \\
& -\left(\left(\tilde{\boldsymbol{B}}-\tilde{\boldsymbol{B}}_{h}\right)\left(\boldsymbol{u}+\boldsymbol{u}_{h}\right), \boldsymbol{u}-\boldsymbol{u}_{h}\right)+\left(\left(\tilde{\boldsymbol{B}}-\tilde{\boldsymbol{B}}_{h}\right) \boldsymbol{u}, \boldsymbol{u}\right) .
\end{aligned}
$$

Note that eigenvector convergence implies that

$$
\left\|\boldsymbol{u}-\boldsymbol{u}_{h}\right\| \leq C h .
$$

In addition, $\left\|\tilde{\boldsymbol{B}}-\tilde{\boldsymbol{B}}_{h}\right\| \leq C h$, so it will be enough to get a higher order bound for the term $\left(\left(\tilde{B}-\tilde{\boldsymbol{B}}_{h}\right) \boldsymbol{u}, \boldsymbol{u}\right)$.

Let $\boldsymbol{x}_{1}=\boldsymbol{S}_{1} \boldsymbol{e}$ and $\boldsymbol{x}_{h, 1}=\boldsymbol{S}_{h, 1} \boldsymbol{e}$. Then $\left(\left(\tilde{\boldsymbol{B}}-\tilde{\boldsymbol{B}}_{h}\right) \boldsymbol{u}, \boldsymbol{u}\right)=2\left(\boldsymbol{x}_{1}-\boldsymbol{x}_{h, 1}, \tilde{\boldsymbol{h}}\right)$ where $\tilde{\boldsymbol{h}}=\tau^{-1} \boldsymbol{S}_{1} \boldsymbol{e}=\tau \nabla \times \boldsymbol{e}$. 
Introduce $\boldsymbol{B}_{h, 1}^{V}$ as the map of $\boldsymbol{X}_{h}$ into $\boldsymbol{V}_{h, 1}^{*}$ defined by

$$
\left\langle\boldsymbol{B}_{h, 1}^{V} \boldsymbol{x}, \boldsymbol{v}_{h}\right\rangle=\left(\boldsymbol{x}, \nabla \times \boldsymbol{v}_{h}\right) \quad \text { for all } \boldsymbol{v}_{h} \in \boldsymbol{V}_{h, 1} .
$$

Similarly, let $\boldsymbol{T}_{h, 1}^{V}: \boldsymbol{V}_{h, 1}^{*} \mapsto \boldsymbol{V}_{h, 1}$ be defined by

$$
\left(\nabla \boldsymbol{T}_{h, 1}^{V} \ell, \nabla \boldsymbol{v}_{h}\right)=\left\langle\ell, \boldsymbol{v}_{h}\right\rangle \quad \text { for all } \boldsymbol{v}_{h} \in \boldsymbol{V}_{h, 1} .
$$

We assume that $\boldsymbol{T}_{h, 1}^{V}$ is used to define the $\boldsymbol{V}_{h, 1}$ component in the definition of $\boldsymbol{S}_{h, 1}$. It follows that for any $\boldsymbol{v}_{h} \in \boldsymbol{X}_{h}, \boldsymbol{T}_{h, 1} \boldsymbol{B}_{h, 1} \boldsymbol{v}_{h}$ consists of two components $\boldsymbol{T}_{h, 1}^{V} \boldsymbol{B}_{h, 1}^{V} v_{h}$ and $T_{h, 1}^{H} B_{h, 1}^{H} v_{h}$, where $T_{h, 1}^{H}$ is the $H_{1}$ part of $\boldsymbol{T}_{h, 1}$ and $B_{h, 1}^{H}$ is the $H_{1}$ part or $\boldsymbol{B}_{h, 1}$, i.e.,

$$
\left\langle B_{h, 1}^{H} \boldsymbol{x}, h_{h}\right\rangle=\left(\boldsymbol{x}, \nabla h_{h}\right) \quad \text { for all } h_{h} \in H_{h, 1} .
$$

The definition of $\boldsymbol{x}_{h, 1}$ states that

$\left(\boldsymbol{x}_{h, 1}, \nabla \times \boldsymbol{T}_{h, 1}^{V} \boldsymbol{B}_{h, 1}^{V} \boldsymbol{v}_{h}\right)+\left(\boldsymbol{x}_{h, 1}, \nabla T_{h, 1}^{H} B_{h, 1}^{H} \boldsymbol{v}_{h}\right)=\left(\boldsymbol{e}, \boldsymbol{T}_{h, 1}^{V} \boldsymbol{B}_{h, 1}^{V} \boldsymbol{v}_{h}\right)$ for all $\boldsymbol{v}_{h} \in \boldsymbol{X}_{h}$.

Note that we used the fact that $\boldsymbol{Q}_{h, 1} \boldsymbol{e}=\mathbf{0}$ above.

Using (4.7), the definition of $\boldsymbol{x}_{1}$ and (4.5) gives

$$
\begin{gathered}
\left(\boldsymbol{x}_{1}-\boldsymbol{x}_{h, 1}, \tilde{\boldsymbol{h}}\right)=\tau\left(\boldsymbol{x}_{1}-\boldsymbol{x}_{h, 1}, \nabla \times \boldsymbol{T}_{1} \nabla \times \boldsymbol{v}-\nabla \times \boldsymbol{T}_{h, 1}^{V} \boldsymbol{B}_{h, 1}^{V} \boldsymbol{v}_{h}\right) \\
-\tau\left(\boldsymbol{x}_{1}-\boldsymbol{x}_{h, 1}, \nabla T_{h, 1}^{V} B_{h, 1}^{V} \boldsymbol{v}_{h}\right)
\end{gathered}
$$

for any $\boldsymbol{v}_{h} \in \boldsymbol{X}_{h}$. The first term in (4.8) can be estimated by

$$
C h\|\boldsymbol{e}\|\left\{\left\|\boldsymbol{T}_{h, 1}^{V}\left(\nabla \times \boldsymbol{v}-\boldsymbol{B}_{h, 1}^{V} \boldsymbol{v}_{h}\right)\right\|_{1}+\left\|\left(\boldsymbol{T}_{1}-\boldsymbol{T}_{h, 1}^{V}\right) \nabla \times \boldsymbol{v}\right\|_{1}\right\} .
$$

We then have

$$
\begin{aligned}
\left\|\boldsymbol{T}_{h, 1}^{V}\left(\nabla \times \boldsymbol{v}-\boldsymbol{B}_{h, 1}^{V} \boldsymbol{v}_{h}\right)\right\|_{1} & \leq \sup _{\boldsymbol{\phi} \in \boldsymbol{V} h, 1} \frac{\left\langle\nabla \times \boldsymbol{v}-\boldsymbol{B}_{h, 1}^{V} \boldsymbol{v}_{h}, \boldsymbol{\phi}\right\rangle}{\|\boldsymbol{\phi}\|_{1}}=\sup _{\boldsymbol{\phi} \in \boldsymbol{V} h, 1} \frac{\left(\boldsymbol{v}-\boldsymbol{v}_{h}, \nabla \times \boldsymbol{\phi}\right)}{\|\boldsymbol{\phi}\|_{1}} \\
& \leq \inf _{\boldsymbol{v}_{h} \in \boldsymbol{X}_{h}}\left\|\boldsymbol{v}-\boldsymbol{v}_{h}\right\| \leq C h^{1-\varepsilon}\|\boldsymbol{v}\|_{1-\varepsilon}
\end{aligned}
$$

and

$$
\left\|\left(\boldsymbol{T}_{1}-\boldsymbol{T}_{h, 1}^{V}\right) \nabla \times \boldsymbol{v}\right\|_{1} \leq C h^{1-\varepsilon}\|\nabla \times \boldsymbol{v}\|_{-\varepsilon} \leq C h^{1-\varepsilon}\|\boldsymbol{v}\|_{1-\varepsilon} .
$$

Finally, the second term in (4.8) is the same as

$$
\begin{aligned}
\tau\left(\boldsymbol{x}_{1}-\boldsymbol{x}_{h, 1}, \nabla T_{h, 1}^{V}\left(\nabla \cdot \boldsymbol{v}-B_{h, 1}^{V} \boldsymbol{v}_{h}\right)\right) & \leq C h \sup _{\phi \in H_{h, 1}} \frac{\left(\left(\nabla \cdot \boldsymbol{v}-B_{h, 1}^{V} \boldsymbol{v}_{h}\right), \phi\right)}{\|\phi\|_{1}} \\
& =C h \sup _{\phi \in H_{h, 1}} \frac{\left(\boldsymbol{v}-\boldsymbol{v}_{h}, \nabla \phi\right)}{\|\phi\|_{1}} \leq C h^{2-\varepsilon}\|\boldsymbol{v}\|_{1-\varepsilon} .
\end{aligned}
$$

Combining the above results, we conclude that $\left|\left(\boldsymbol{x}_{1}-\boldsymbol{x}_{h, 1}, \boldsymbol{h}\right)\right| \leq C(\tau) h^{2-\varepsilon}$ for any $0<\varepsilon<\frac{1}{2}$ and, therefore, we proved the following improved convergence estimate.

Theorem 4.3. Assume that $\Omega$ is a convex polyhedron, $\epsilon=\mu=1, \boldsymbol{T}_{h, 1}$ is defined in terms of the direct solve (4.6), and the eigenvectors are such that $\boldsymbol{e} \cdot \boldsymbol{n} \in H^{3 / 2}(\Gamma)$ for each face $\Gamma$ of $\partial \Omega$.

Let $\lambda=i \omega$ be a fixed eigenvalue of (2.1), $\tau^{2}=\omega^{-2}$, and $\left\{\tau_{i}^{2}(h)\right\}_{i=1}^{k}$ be the eigenvalues of $\boldsymbol{S}_{h, 1}^{*} \boldsymbol{S}_{h, 1}$ that are an approximation of $\tau^{2}$. Fix $0<\varepsilon<\frac{1}{2}$. Then, there exists a positive constant $C=C(\lambda)$ independent of $h$ such that for all $i=$ $1, \ldots, k$,

$$
\left|\tau^{2}-\tau_{i}^{2}(h)\right| \leq C h^{2-\varepsilon} .
$$




\section{The General DOMAIN CASE}

In this section, we discuss the modifications necessary to deal with nonsimply connected domains and domains with multiply connected boundary components. The only essential difference is that we shall have to increase the spaces $H_{1}$ and $H_{2}$ used above with an analogous increase in their discrete counterparts.

Our assumptions on the topology of the domains are identical to those in 2 (see, e.g., 2, Hypothesis 3.3]). This allows the introduction of cutting surfaces $\Sigma_{j}, j=1, \ldots, J$ so that the domain $\Omega_{0}=\Omega \backslash\left(\bigcup \Sigma_{j}\right)$ is simply connected. We also denote the connected components of $\partial \Omega$ by $\Gamma_{i}, i=0, \ldots, I$ with $\Gamma_{0}$ being the outermost boundary. There are two finite-dimensional spaces associated with the above.

First, we define $W_{n}$ to be the space spanned by the functions $\left\{\zeta_{j}\right\}$ satisfying

$$
\begin{aligned}
-\nabla \cdot \mu \nabla \zeta_{j} & =0 \text { on } \Omega_{0}, \\
\mu \frac{\partial \zeta_{j}}{\partial \boldsymbol{n}} & =0 \text { on } \partial \Omega, \\
{\left[\zeta_{j}\right]_{i}=\delta_{i j} } & \text { and }\left[\mu \frac{\partial \zeta_{j}}{\partial \boldsymbol{n}}\right]_{i}=0 \text { on } \Sigma_{i} .
\end{aligned}
$$

Here []$_{i}$ denotes the jump across $\Sigma_{i}$ and $\delta_{i j}$ denotes the Kronecker delta. Let

$$
\Theta=\left\{\phi \in H^{1}\left(\Omega_{0}\right):[\phi]_{j}=\text { constant }, j=1, \ldots J\right\} .
$$

The solution $\zeta_{j}$ (up to an additive constant) of the above problem is uniquely determined in $\Theta$. Indeed, if $\zeta_{j}^{0}$ satisfies $\left[\zeta_{j}^{0}\right]_{i}=\delta_{i j}$, then $\zeta_{j}=\zeta_{j}^{0}+\zeta_{j}^{1}$ where $\zeta_{j}^{1}$ solves

$$
\left(\mu \zeta_{j}^{1}, \phi\right)=-\left(\mu \zeta_{j}^{0}, \phi\right)
$$

for all $\phi \in H^{1}(\Omega)$. Let $\widetilde{\nabla} \zeta_{j}$ denote the distributional gradient of $\zeta_{j}$ with respect to $\Omega_{0}$. It follows from $\left[\zeta_{j}\right]=\delta_{i j}$ on $\Sigma_{i}$ that $\widetilde{\nabla} \zeta_{j}$ is in $\boldsymbol{H}$ (curl) (cf., [2, Lemma 3.11]) and $(\boldsymbol{e}, \boldsymbol{h}) \equiv\left(0, \widetilde{\nabla} \zeta_{j}\right)$ satisfies (2.1) with $\lambda=0$.

The second finite-dimensional space is defined as follows. For each $i=1, \ldots, I$, we define $\psi_{i}$ to be the function in $H^{1}(\Omega)$ satisfying

$$
\begin{aligned}
-\nabla \cdot \epsilon \nabla \psi_{i} & =0, \quad \text { in } \Omega, \\
\psi_{i} & =\delta_{i j}, \quad \text { on } \Gamma_{j},
\end{aligned}
$$

and we set $W_{d}$ to be the span of $\left\{\psi_{i}\right\}$. We note that $\nabla \psi$ is in $\boldsymbol{H}_{0}(\mathbf{c u r l})$ and $(\boldsymbol{e}, \boldsymbol{h}) \equiv\left(\nabla \psi_{i}, \mathbf{0}\right)$ satisfies (2.1) with $\lambda=0$.

As discussed in [12, for the inf-sup condition (2.9) to hold in the case of nonsimply connected domains, we need to increase the space $H_{1}$, specifically, we set $H_{1}=H^{1}(\Omega) \oplus W_{n}$ and replace $\nabla h$ in the definition of $b_{1}$ with $\widetilde{\nabla} h$. In the case of multiply connected boundary components, we set $H_{2}=H_{0}^{1}(\Omega) \oplus W_{d}$.

The analysis in Section 2 still goes through with these definitions. Let $(\boldsymbol{e}, \boldsymbol{h})$ satisfy (2.1) with nonzero $\lambda$. Then, by (2.3),

$$
0=\left(\nabla \times \boldsymbol{e}, \widetilde{\nabla} \zeta_{j}\right)=-\lambda\left(\mu \boldsymbol{h}, \widetilde{\nabla} \zeta_{j}\right) .
$$

Similarly,

$$
0=\left(\nabla \times \boldsymbol{h}, \nabla \psi_{i}\right)=\lambda\left(\epsilon \boldsymbol{e}, \nabla \psi_{i}\right) .
$$

Thus, the eigenvectors of (2.11) with nonzero eigenvalues still satisfy (2.11) with $\boldsymbol{S}_{k}$ defined with $H_{k}$ as above and Theorem 2.1 holds as well. 
We next consider discretization. Without loss of generality, we may assume that the cuts $\left\{\Sigma_{i}\right\}$ align with the mesh. It is easy to see that $H_{1}$ and $\Theta$ coincide. Thus, to define $H_{h, 1}$, we start with the usual approximation space for $H^{1}(\Omega)$ and append functions which are discontinuous on the cuts. Specifically, we add basis functions which are one on the nodes on one side of $\Sigma_{i}$ and vanish on all remaining nodes (including those on the opposite side of $\Sigma_{i}$ ).

The discrete inf-sup condition still holds. Indeed, the strategy employed in [12] proceeds as follows. The continuous inf-sup condition implies that for $\boldsymbol{x} \in \boldsymbol{X}_{h}$, there is a $(\boldsymbol{v}, h) \in \boldsymbol{Y}_{1}$ satisfying

$$
\|\boldsymbol{x}\|_{\boldsymbol{L}^{2}(\Omega)} \leq C \frac{b_{1}(\boldsymbol{x},(\boldsymbol{v}, h))}{\|(\boldsymbol{v}, h)\|_{\boldsymbol{Y}_{1}}} .
$$

One then constructs a pair $\left(\boldsymbol{v}_{h}, h_{h}\right) \in \boldsymbol{Y}_{k, h}$ satisfying

$$
b_{1}\left(\boldsymbol{x},\left(\boldsymbol{v}_{h}, h_{h}\right)=b_{1}(\boldsymbol{x},(\boldsymbol{v}, h))\right.
$$

and

$$
\left\|\left(\boldsymbol{v}_{h}, h_{h}\right)\right\|_{\boldsymbol{Y}_{1}} \leq C\|(\boldsymbol{v}, h)\|_{\boldsymbol{Y}_{1}} .
$$

The construction proceeds by first using a stable approximation operator $I_{h}$ as an initial approximation and using the bubble functions to enforce (5.1) on the remainder (see [12]). We simply use a modified approximation operator for $H_{1}$. Specifically, let $\tilde{I}_{h}$ be a stable approximation operator into the subspace of piecewise linear functions with arbitrary discontinuities across the cuts and define $I_{h} h$ equal to $\tilde{I}_{h} h$ on the nodes not on the cut and by a boundary averaging operator (on each side of the cut) such as that given in 37] to define the nodes on the cut. This results in a stable approximation operator. Moreover, since $h$ differs by a constant on each side of the cut and the boundary averaging operator preserves constants, $I_{h} h$ is in $H_{h, 1}$ and has the same jumps as $h$. Using $I_{h}$, the remainder of the proof in 12 goes through.

We note that

$$
H_{2}=\left\{\phi \in H^{1}(\Omega):\left.\phi\right|_{\Gamma_{i}}=\text { constant }\right\}
$$

In this case, we start with the finite-element approximation to $H_{0}^{1}(\Omega)$ and append basis functions which are one on a given connected component of the boundary and vanish at all remaining nodes. To prove the discrete inf-sup condition, we are again left with the construction of a suitable stable approximation operator. If $\tilde{I}_{h}$ denotes a stable approximation operator into the finite-element subspace with arbitrary boundary values, we set $I_{h} h$ to be $\tilde{I}_{h} h$ at the interior nodes and interpolate $h$ at the boundary nodes. It is easy to prove that $I_{h}$ is a stable interpolation operator which reproduces $h$ on $\partial \Omega$. With this operator the proof in [12] carries over.

\section{NumERICAL EXPERIMENTS}

In this section we report results from some numerical experiments with the leastsquares method for the problem (2.15). We concentrate on the case of a simply connected polyhedral domain in $\mathbb{R}^{3}$.

The eigenvalues $\left\{\lambda_{i}\right\}$ of (2.1) are purely imaginary and $\left\{\left|\lambda_{i}\right|^{2}\right\}=\left\{\omega_{i}^{2}\right\}$ where $\left\{\omega_{i}^{2}\right\}$ are the eigenvalues of the curl-curl operator, which are customarily reported in the literature. Specifically, $\left\{\omega_{i}^{2}\right\}$ are real, nonnegative, and satisfy $\nabla \times \mu^{-1} \nabla \times \boldsymbol{e}=$ $\omega^{2} \epsilon \boldsymbol{e}, \nabla \cdot \epsilon \boldsymbol{e}=0$, with boundary conditions $\boldsymbol{e} \times \boldsymbol{n}=\mathbf{0}$. Our analysis implies that $\omega_{i}^{2}=\tau_{i}^{-2}$. 
We report computations involving both tetrahedral and hexahedral meshes. Although there are many analyses available for tetrahedral meshes using methods based on curl conforming finite-element approximations [6, 29, 32, 31, very little has been done for general hexahedral meshes. In contrast, the analysis presented in this paper easily extends to general hexahedral meshes.

The outline of our computational approach is as follows. We start with a coarse mesh and apply few levels of uniform refinement. We use piecewise constant vector functions for the space $\boldsymbol{X}_{h}$ and piecewise linear plus face bubble vector functions for each component of $\boldsymbol{Y}_{h, k}$. These are described in more detail in [12, where it is shown that such a pair of spaces satisfy the inf-sup condition (3.1). On each mesh level we compute a number of the largest eigenvalues and corresponding eigenfunctions of $\boldsymbol{S}_{h, 1}^{*} \boldsymbol{S}_{h, 1}$ using a slightly modified version of the Locally Optimal Block Preconditioned Conjugate Gradient Method (LOBPCG), (see [28]). The actions of $\boldsymbol{T}_{h, k}$ for $k=1,2$ are implemented using a two-level algorithm involving a GaussSeidel sweep over the bubble functions and a V-cycle multigrid preconditioner for the remaining piecewise linear functions. We take $\epsilon=\mu=1$.

The first test problem is posed on the unit cube partitioned into a uniform tetrahedral mesh. The eigenvalues and eigenfunctions of this problem can be computed exactly. The eigenfunctions are tensor products of trigonometric functions. Eigenvalues are of the form $\left\{\tau_{i}^{2}\right\}=\left\{\frac{1}{k \pi^{2}}\right\}$, where $k=k_{1}^{2}+k_{2}^{2}+k_{3}^{2}$ and $\left\{k_{i}\right\}_{i=1}^{3}$ are nonnegative integers satisfying $k_{1} k_{2}+k_{2} k_{3}+k_{3} k_{1}>0$. Triplets with $k_{1} k_{2} k_{3}>0$ generate two linearly independent eigenfunctions.

Figure 1 gives the eigenvalues of $\boldsymbol{S}_{h, 1}^{*} \boldsymbol{S}_{h, 1}$ (approximating those of $\boldsymbol{S}_{2} \boldsymbol{S}_{1}$ ) as a function of the number of mesh refinement levels. Observe that the method performs well with multiple eigenvalues. In addition, the eigenvalue convergence appears to be monotone.

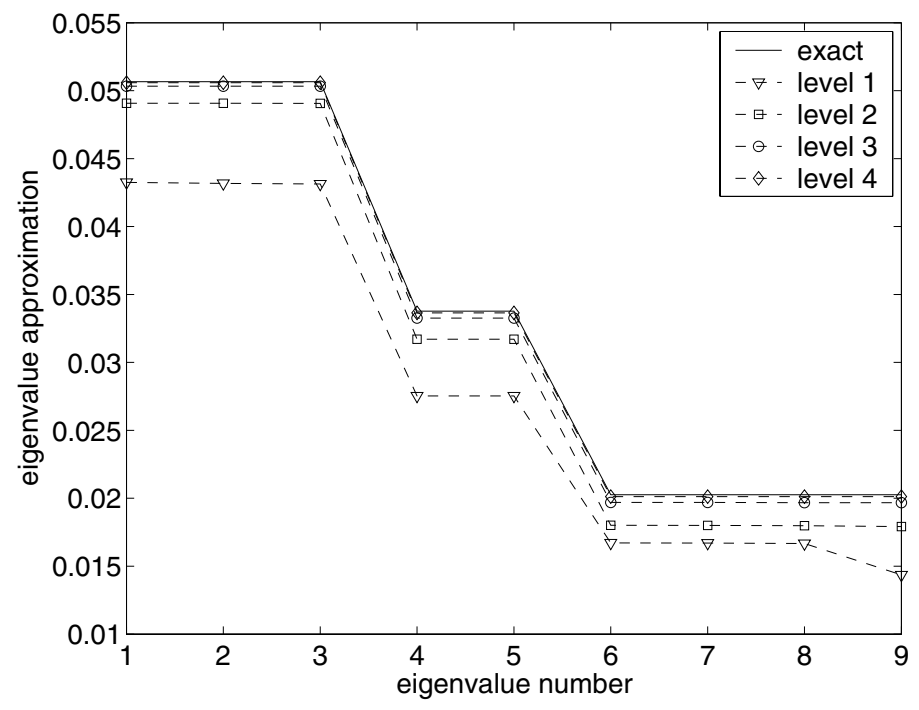

Figure 1. Unit cube: eigenvalue convergence 

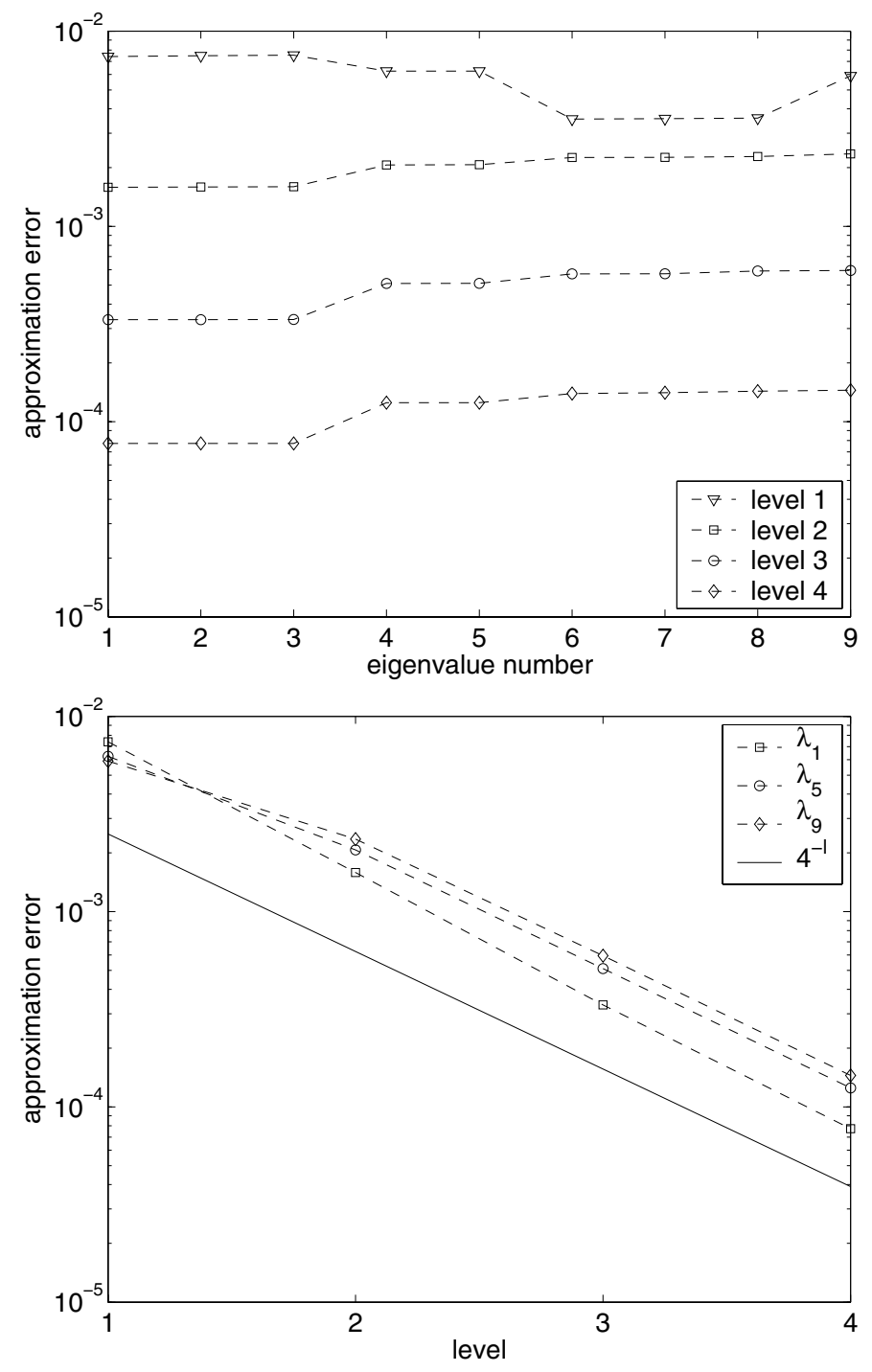

FiguRe 2. Unit cube: approximation error

Figure 2 presents the same results in different formats. On the top we show the approximation in the error for each $\left\{\tau_{i}^{2}\right\}$. We note that the approximation becomes slightly worse with the increase of the eigenvalue number. This is further examined on the bottom,where we are looking at the error in three representative eigenvalues, $\tau_{1}^{2}, \tau_{5}^{2}$ and $\tau_{9}^{2}$, on the different levels of approximation. As expected, (see Section 4) we have almost quadratic convergence for the eigenvalues.

Our second example is the computation of the eigenmodes of the unit sphere. The eigenvalues and eigenfunctions are known, but they are not as simple as in the previous test. 
TABLE 6.1. Unit sphere: exact eigenvalues

\begin{tabular}{|c|c|c|}
\hline$i$ & $\omega_{i}^{2}$ & type \\
\hline 1 & $7.5279 \mathrm{e}+00$ & TM $\left(\hat{\omega}_{11}^{2}\right)$ \\
2 & $1.4979 \mathrm{e}+01$ & TM $\left(\hat{\omega}_{21}^{2}\right)$ \\
3 & $2.0191 \mathrm{e}+01$ & TE $\left(\omega_{11}^{2}\right)$ \\
4 & $2.4735 \mathrm{e}+01$ & TM $\left(\hat{\omega}_{31}^{2}\right)$ \\
5 & $3.3217 \mathrm{e}+01$ & TE $\left(\hat{\omega}_{21}^{2}\right)$ \\
6 & $3.6747 \mathrm{e}+01$ & TM $\left(\hat{\omega}_{41}^{2}\right)$ \\
7 & $3.7415 \mathrm{e}+01$ & TM $\left(\hat{\omega}_{12}^{2}\right)$ \\
\hline
\end{tabular}

Specifically, the eigenvalues $\left\{\omega_{i}^{2}\right\}=\left\{\omega_{m n}^{2}, \hat{\omega}_{m n}^{2}: m, n=1,2, \ldots\right\}$ are split into two groups:

- Transverse Electric (TE), which satisfy

$$
j_{m}\left(\omega_{m n}^{2}\right)=0,
$$

and

- Transverse Magnetic (TM), which satisfy

$$
j_{m}\left(\hat{\omega}_{m n}^{2}\right)+\hat{\omega}_{m n}^{2} j_{m}^{\prime}\left(\hat{\omega}_{m n}^{2}\right)=0 .
$$

Here $j_{m}$ is the $m$ th order spherical Bessel function and $j_{m}^{\prime}$ is its derivative. The numerical values for the first few of them (without accounting for their multiplicity) are given in Table 6.1.

We used a set of hexahedral meshes starting with the coarse mesh shown in Figure 3. Their characteristics together with the number of iterations of the eigensolver are given in Table 6.2.

TABLE 6.2. Unit sphere: test meshes and number of LOBPCG iterations

\begin{tabular}{|c|c|c|c|c|c|c|}
\hline level & $h_{\min }$ & $h_{\max }$ & \#vertices & \#faces & \#elements & $n_{i t}$ \\
\hline 1 & 0.109665 & 0.255241 & 976 & 2700 & 875 & 22 \\
2 & 0.046295 & 0.124278 & 9736 & 28314 & 9317 & 13 \\
3 & 0.023515 & 0.066545 & 66256 & 195804 & 64827 & 13 \\
\hline
\end{tabular}

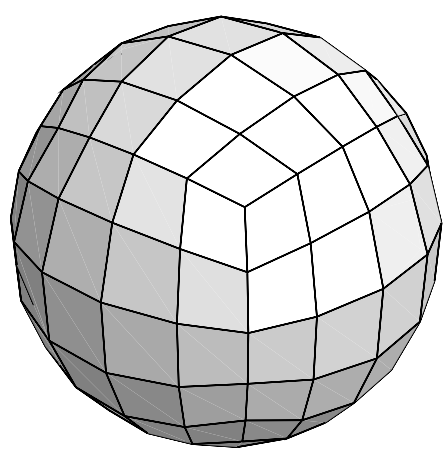

Figure 3. Unit sphere: initial mesh 

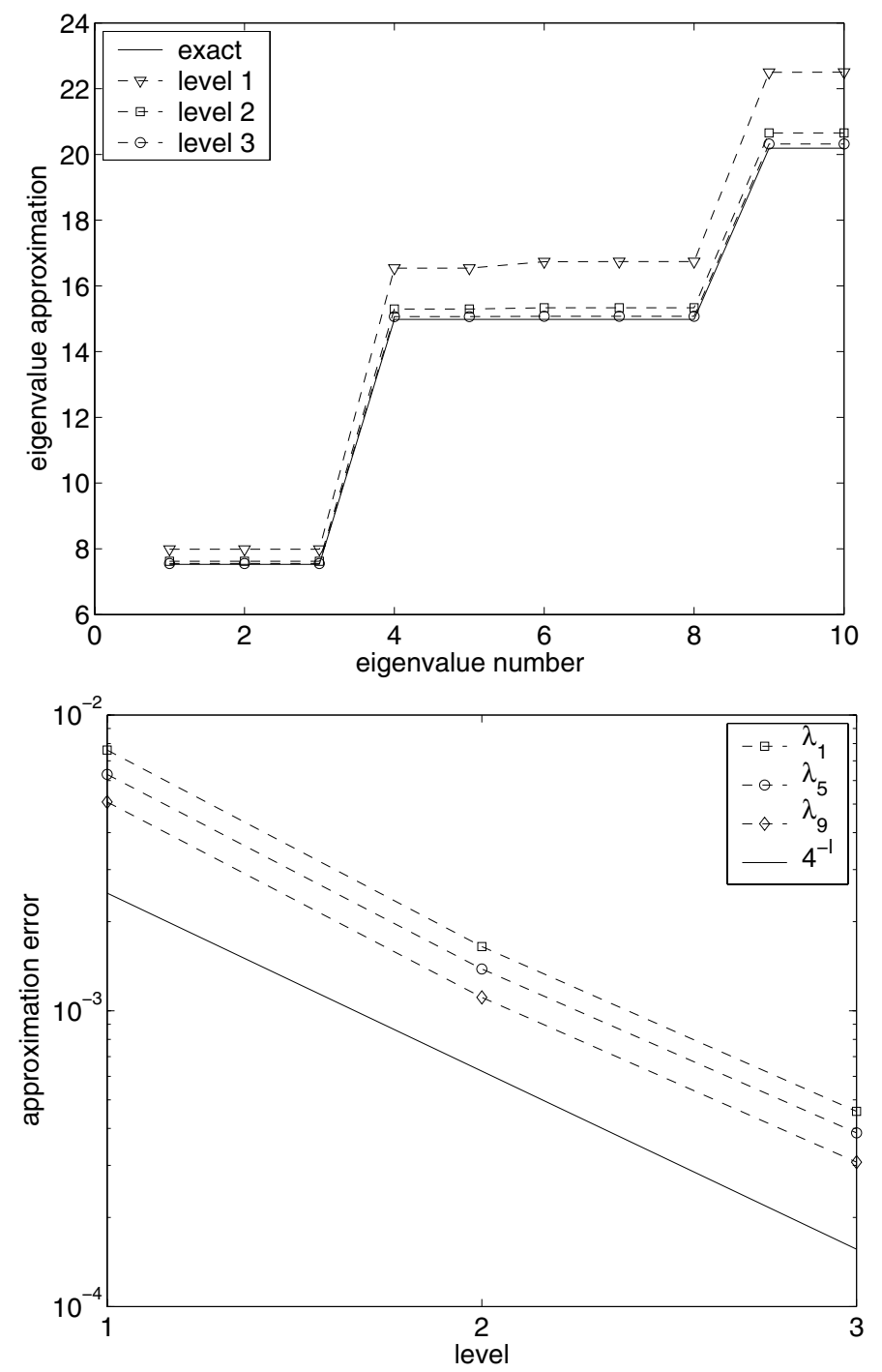

FIGURE 4. Unit sphere: eigenvalue convergence

We proceeded to compute the first ten eigenfunctions. The approximation errors for the eigenvalues of (2.1) and $\boldsymbol{S}_{h, 1}^{*} \boldsymbol{S}_{h, 1}$ are presented in Figure 4. The results are similar to the previous test problem.

Our third example is the computation of the eigenvalues in the Fichera corner $[-1,1]^{3} \backslash[-1,0]^{3}$. The exact eigenfunctions are not known, but some of them have singularities at the origin which makes the problem difficult to approximate. We will compare our results with the ones from Table 6.3 which are taken from M. Dauge's benchmark website [16].

Two tests were performed for this problem using unstructured tetrahedral and uniform hexahedral meshes. The initial meshes are shown in Figure 5. The compu- 
TABLE 6.3. Fichera corner, benchmark results from [16]

\begin{tabular}{|c|c|c|c|}
\hline$i$ & $\omega_{i}^{2}$ & reliable digits & conjectured eigenvalue \\
\hline 1 & $3.31381 \mathrm{e}+00$ & 1 & $3.2 ? ? ? \mathrm{e}+00$ \\
2 & $5.88635 \mathrm{e}+00$ & 3 & $5.88 ? ? \mathrm{e}+00$ \\
3 & $5.88635 \mathrm{e}+00$ & 3 & $5.88 ? ? \mathrm{e}+00$ \\
4 & $1.06945 \mathrm{e}+01$ & 4 & $1.0694 \mathrm{e}+01$ \\
5 & $1.06945 \mathrm{e}+01$ & 4 & $1.0694 \mathrm{e}+01$ \\
6 & $1.07006 \mathrm{e}+01$ & 2 & $1.07 ? ? \mathrm{e}+01$ \\
7 & $1.23345 \mathrm{e}+01$ & 3 & $1.232 ? \mathrm{e}+01$ \\
8 & $1.23345 \mathrm{e}+01$ & 3 & $1.232 ? \mathrm{e}+01$ \\
\hline
\end{tabular}
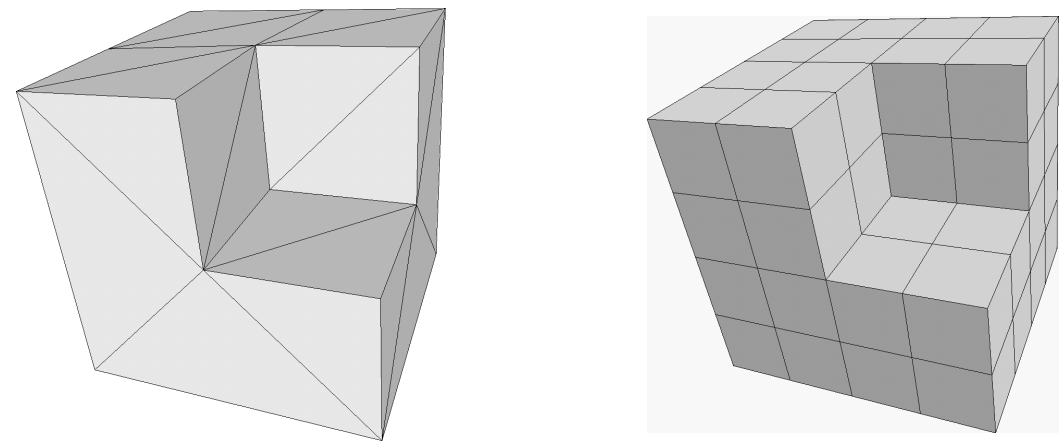

FIGURE 5. Fichera corner: initial meshes

tations were performed on refined grids consisting of 28489 vertices, 323072 faces and 159744 tetrahedra and 31841 vertices, 89088 faces and 28672 hexahedra, respectively.

The results of the eigenvalue approximations for the first eight eigenfunctions in each case are reported on Table 6.4.

We note that the hexahedral mesh offers comparable approximation with significantly less memory usage. This can be explained by the fact that the mesh is uniform and that the dimensions of $\boldsymbol{X}_{h}$ and $\boldsymbol{Y}_{h, k}$ are balanced better in this case.

Our final problem involves complicated geometry modeled with fine hexahedral mesh. It is a linear accelerator induction cell taken from Lawrence Livermore

TABLE 6.4. Fichera corner: results for tetrahedral mesh (columns 2 and 3 ) and hexahedral mesh (column 4)

\begin{tabular}{|c|c|c|c|}
\hline$i$ & $\omega_{h, i}^{2}$ & $\left|\omega_{i}^{2}-\omega_{h, i}^{2}\right|$ & $\left|\omega_{i}^{2}-\omega_{h, i}^{2}\right|$ \\
\hline 1 & $3.23432 \mathrm{e}+00$ & $7.94855 \mathrm{e}-02$ & $2.63062 \mathrm{e}-02$ \\
2 & $5.88267 \mathrm{e}+00$ & $3.67742 \mathrm{e}-03$ & $1.69117 \mathrm{e}-02$ \\
3 & $5.88371 \mathrm{e}+00$ & $2.64462 \mathrm{e}-03$ & $1.69511 \mathrm{e}-02$ \\
4 & $1.06789 \mathrm{e}+01$ & $1.55709 \mathrm{e}-02$ & $6.22111 \mathrm{e}-02$ \\
5 & $1.06832 \mathrm{e}+01$ & $1.12777 \mathrm{e}-02$ & $6.22377 \mathrm{e}-02$ \\
6 & $1.06945 \mathrm{e}+01$ & $6.08114 \mathrm{e}-03$ & $1.03244 \mathrm{e}-01$ \\
7 & $1.23653 \mathrm{e}+01$ & $3.07189 \mathrm{e}-02$ & $1.20678 \mathrm{e}-01$ \\
8 & $1.23723 \mathrm{e}+01$ & $3.77137 \mathrm{e}-02$ & $1.22141 \mathrm{e}-01$ \\
\hline
\end{tabular}





FiguRE 6. Accelerator induction cell: eigenfunctions 1,2, 4 and 8

National Laboratory's EMSolve project; see [1. The mesh has 46382 vertices, 128992 faces, and 41344 elements and comes form a real-world application.

Our code successfully computed the first ten eigenvalues of this difficult problem. Some of the computed eigenfunctions are shown in Figure 6 .

Finally, we want to mention that further numerical experiments seem to suggest that the use of the projectors $\boldsymbol{Q}_{h, k}$ and the stabilizing face bubble functions are essential for the convergence and cannot be avoided.

In conclusion, the experiments show that the new method performs quite well in a variety of applications. Let us stress again that spurious eigenmodes are completely avoided. We also conclude that LOBPCG seems to be a good choice for eigensolver, yielding a constant number of iterations in the tests presented.

\section{Appendix}

In this appendix, we will provide a regularity estimate for the solution of (4.4). Assume that the domain $\Omega$ is convex. Let $\boldsymbol{f} \in \boldsymbol{H}^{-1}(\Omega)$ and $\nabla \cdot \boldsymbol{f}=0$ in the sense of distributions. As discussed earlier, the div-curl system

$$
\begin{aligned}
& \nabla \times \boldsymbol{x}=\boldsymbol{f} \text { in } \Omega, \\
& \nabla \cdot \boldsymbol{x}=0 \text { in } \Omega \text {, } \\
& \boldsymbol{x} \cdot \boldsymbol{n}=0 \text { on } \partial \Omega
\end{aligned}
$$

has a unique "weak" solution $\boldsymbol{x} \in \boldsymbol{L}^{2}(\Omega)$ satisfying

$$
b_{1}(\boldsymbol{x},(\boldsymbol{v}, h))=\langle\boldsymbol{f}, \boldsymbol{v}\rangle \quad \text { for all }(\boldsymbol{v}, h) \in \boldsymbol{Y}_{1} .
$$

For $\varepsilon \in[0,1]$, define $H_{0}^{\varepsilon}(\Omega)=\left[L_{2}(\Omega), H_{0}^{1}(\Omega)\right]_{\varepsilon}$ - the interpolation space between $L_{2}(\Omega)$ and $H_{0}^{1}(\Omega)$. Recall that $H^{-\varepsilon}(\Omega)$ is the dual of $H_{0}^{\varepsilon}(\Omega)$, and $\boldsymbol{H}^{-\varepsilon}(\Omega)=$ $\left(H^{-\varepsilon}(\Omega)\right)^{3}$.

For $\boldsymbol{g} \in\left(\boldsymbol{H}^{\varepsilon}(\Omega)\right)^{*}$, consider the problem of finding $\psi \in H_{0}^{1-\varepsilon}(\Omega)$ such that

$$
\langle-\Delta \theta, \psi\rangle=\langle\boldsymbol{g}, \nabla \theta\rangle \quad \text { for all } \theta \in H_{0}^{1}(\Omega) \cap H^{1+\varepsilon}(\Omega) .
$$

This problem has a unique solution since the operator $-\Delta$ defines an isomorphism of $H_{0}^{1}(\Omega) \cap H^{1+\varepsilon}(\Omega)$ onto $H^{-1+\varepsilon}(\Omega)$. Additionally, we have

$$
\|\nabla \psi\|_{\boldsymbol{H}^{-\varepsilon}(\Omega)} \leq\|\psi\|_{H_{0}^{1-\varepsilon}(\Omega)} \leq C\|\boldsymbol{g}\|_{\left(\boldsymbol{H}^{\varepsilon}(\Omega)\right)^{*}},
$$

and therefore by setting $\boldsymbol{Q}_{1} \boldsymbol{g}=\nabla \psi$ we get the unique continuous extension of $\boldsymbol{Q}_{1}$ as an operator from $\left(\boldsymbol{H}^{\varepsilon}(\Omega)\right)^{*}$ to $\boldsymbol{H}^{-\varepsilon}(\Omega)$. Thus, in particular,

$$
\left\|\boldsymbol{Q}_{1} \boldsymbol{g}\right\|_{\boldsymbol{H}^{-1}(\Omega)} \leq C\|\boldsymbol{g}\|_{\left(\boldsymbol{H}^{1}(\Omega)\right)^{*}}
$$


Analogous to the definition in $\S 2$, let $\boldsymbol{S}_{1}$ be the solution operator defined as $\boldsymbol{S}_{1} \boldsymbol{g}=\boldsymbol{x}$, where $\boldsymbol{x}$ solves (7.2) with data $\boldsymbol{f}=\left(\boldsymbol{I}-\boldsymbol{Q}_{1}\right) \boldsymbol{g}$.

Lemma 7.1. For any $\varepsilon \in[0,1], \boldsymbol{S}_{1}:\left(\boldsymbol{H}^{\varepsilon}(\Omega)\right)^{*} \mapsto \boldsymbol{H}^{1-\varepsilon}(\Omega)$ is a bounded linear operator.

Proof. For any $\boldsymbol{g} \in\left(\boldsymbol{H}^{\varepsilon}(\Omega)\right)^{*}$ we have that $\left(\boldsymbol{I}-\boldsymbol{Q}_{1}\right) \boldsymbol{g} \in \boldsymbol{H}^{-1}(\Omega)$ and

$$
\left\langle\left(\boldsymbol{I}-\boldsymbol{Q}_{1}\right) \boldsymbol{g}, \nabla \theta\right\rangle=0
$$

for arbitrary $\theta \in \mathcal{D}(\Omega)$. Therefore, the compatability condition (2.8) is satisfied, and $\boldsymbol{S}_{1} \boldsymbol{g}$ is well defined. Moreover, the convexity of $\Omega$ and the inf-sup condition (2.9) imply that

$$
\left\|\boldsymbol{S}_{1} \boldsymbol{g}\right\|_{1} \leq C\left\|\left(\boldsymbol{I}-\boldsymbol{Q}_{1}\right) \boldsymbol{g}\right\|_{0} \quad \text { and } \quad\left\|\boldsymbol{S}_{1} \boldsymbol{g}\right\|_{0} \leq C\left\|\left(\boldsymbol{I}-\boldsymbol{Q}_{1}\right) \boldsymbol{g}\right\|_{\boldsymbol{H}^{-1}},
$$

for $\boldsymbol{g} \in \boldsymbol{L}^{2}(\Omega)$ and $\boldsymbol{g} \in\left(\boldsymbol{H}^{1}(\Omega)\right)^{*}$, respectively. Using the boundedness of $\boldsymbol{Q}_{1}$ we get

$$
\left\|\boldsymbol{S}_{1} \boldsymbol{g}\right\|_{1} \leq C\|\boldsymbol{g}\|_{0} \quad \text { and } \quad\left\|\boldsymbol{S}_{1} \boldsymbol{g}\right\|_{0} \leq C\|\boldsymbol{g}\|_{\left(\boldsymbol{H}^{1}(\Omega)\right)^{*}} .
$$

Thus, by interpolation,

$$
\left\|\boldsymbol{S}_{1} \boldsymbol{g}\right\|_{1-\varepsilon} \leq C\|\boldsymbol{g}\|_{\left(\boldsymbol{H}^{\varepsilon}\right)^{*}} \quad \text { for all } \boldsymbol{g} \in\left(\boldsymbol{H}^{\varepsilon}(\Omega)\right)^{*} .
$$

Corollary 7.1. Let $\varepsilon \in\left(0, \frac{1}{2}\right)$. There exists $C=C(\varepsilon)>0$, such that for data $\boldsymbol{f} \in \boldsymbol{H}^{-\varepsilon}(\Omega)$, with $\nabla \cdot \boldsymbol{f}=0$, the solution of $(7.2)$ is in $\boldsymbol{H}^{1-\varepsilon}(\Omega)$ and we have the stability estimate

$$
\|\boldsymbol{x}\|_{1-\varepsilon} \leq C\|\boldsymbol{f}\|_{-\varepsilon} .
$$

Proof. Since $\varepsilon<\frac{1}{2}$, we have $\boldsymbol{H}^{-\varepsilon}(\Omega)=\left(\boldsymbol{H}^{\varepsilon}(\Omega)\right)^{*}$. By (7.3) and the fact that $\mathcal{D}(\Omega)$ is dense in $H_{0}^{1}(\Omega) \cap H^{1+\varepsilon}(\Omega)$, it follows that $\boldsymbol{Q}_{1} \boldsymbol{f}=\mathbf{0}$ when $\boldsymbol{f} \in \boldsymbol{H}^{-\varepsilon}(\Omega)$ and $\nabla \cdot \boldsymbol{f}=0$. For such $\boldsymbol{f}, \boldsymbol{S}_{1} \boldsymbol{f}$ coincides with the solution $\boldsymbol{x}$ of (7.2). The corollary follows from Lemma 7.1 .

Remark 7.1. When $\boldsymbol{f} \in\left(\boldsymbol{H}^{\varepsilon}(\Omega)\right)^{*}$ with $\varepsilon \in\left[\frac{1}{2}, 1\right]$, the condition $\boldsymbol{Q}_{1} \boldsymbol{f}=\mathbf{0}$ implies $\nabla \cdot f=0$ by (7.4). The converse is false. Indeed, for example, let $\varepsilon=1$ and $\phi \in L^{2}(\Omega)$ be a nonconstant harmonic function. Define $\boldsymbol{f} \in\left(\boldsymbol{H}^{1}(\Omega)\right)^{*}$ by

$$
\langle\boldsymbol{f}, \boldsymbol{v}\rangle=-(\phi, \nabla \cdot \boldsymbol{v}) \text { for all } \boldsymbol{v} \in \boldsymbol{H}^{1}(\Omega) .
$$

Clearly $\nabla \cdot \boldsymbol{f}=0$. On the other hand, (17.3) implies that $\boldsymbol{Q}_{1} \boldsymbol{f}=\left.\boldsymbol{f}\right|_{\boldsymbol{H}^{-1}}=\nabla \phi \neq \mathbf{0}$.

\section{REFERENCES}

[1] EMSolve: unstructured grid computational electromagnetics using mixed finite element methods. URL http://www.llnl.gov/CASC/emsolve/.

[2] C. Amrouche, C. Bernardi, M. Dauge, and V. Girault. Vector potentials in three-dimensional non-smooth domains. Math. Methods Appl. Sci., 21(9):823-864, 1998. MR 1626990 (99e:35037)

[3] A. Bermúdez and D. G. Pedreira. Mathematical analysis of a finite element method without spurious solutions for computation of dielectric waveguides. Numer. Math., 61(1):39-57, 1992. MR1145906 (92m:65139)

[4] D. Boffi. Fortin operator and discrete compactness for edge elements. Numer. Math., 87(2):229-246, 2000. MR1804657 (2001k:65168)

[5] D. Boffi, F. Brezzi, and L. Gastaldi. On the convergence of eigenvalues for mixed formulations. Ann. Scuola Norm. Sup. Pisa Cl. Sci. (4), 25(1-2):131-154 (1998), 1997. MR:1655512 (99i:65121) 
[6] D. Boffi, P. Fernandes, L. Gastaldi, and I. Perugia. Computational models of electromagnetic resonators: analysis of edge element approximation. SIAM J. Numer. Anal., 36(4):1264-1290 (electronic), 1999.

[7] A. Bossavit. Computational electromagnetism. Academic Press Inc., San Diego, CA, 1998. Variational formulations, complementarity, edge elements. MR1488417 (99m:78001)

[8] J. Bramble and J. Pasciak. New convergence estimates for multigrid algorithms. Math. Comp., 49:311-329, 1987. MR0906174 (89b:65234)

[9] J. H. Bramble, T. V. Kolev, and J. E. Pasciak. A least-squares method for the time-harmonic Maxwell equations. in preparation, 2004.

[10] J. H. Bramble, D. Y. Kwak, and J. E. Pasciak. Uniform convergence of multigrid $V$-cycle iterations for indefinite and nonsymmetric problems. SIAM J. Numer. Anal., 31(6):17461763, 1994. MR1262601 (95f:65202)

[11] J. H. Bramble and J. E. Osborn. Rate of convergence estimates for nonselfadjoint eigenvalue approximations. Math. Comp., 27(123):525-549, 1973. MR0366029 (51 \#2280)

[12] J. H. Bramble and J. E. Pasciak. A new approximation technique for div-curl systems. Math. Comp., 73(248):1739-1762, 2004. MR2059734 (2005b:65124)

[13] M. Costabel, M. Dauge, and D. Martin. Numerical investigation of a boundary penalization method for Maxwell equations. 1999. Preprint.

[14] M. Costabel, M. Dauge, and D. Martin. Weighted regularization of Maxwell equations in polyhedral domains. 2001. Preprint.

[15] M. Costabel, M. Dauge, and S. Nicaise. Singularities of Maxwell interface problems. M2AN Math. Model. Numer. Anal., 33(3):627-649, 1999. MR1713241(2001g:78005)

[16] M. Dauge. Benchmark computations for maxwell equations for the approximation of highly singular solutions. URL http://perso.univ-rennes1.fr/monique.dauge/benchmax.html.

[17] M. Dauge. Elliptic Boundary Value Problems on Corner Domains. Lecture Notes in Mathematics, 1341, Springer-Verlag, 1988. MR0961439 (91a:35078)

[18] E. D'yakonov and M. Orekhov. Minimization of the computational labor in determining the first eigenvalues of differential operators. Math. Notes, 27:382-391, 1980. MR 0578262 (82a:65086)

[19] C. Emson, J. Simkin, and C. Trowbridge. Further developments in three dimensional eddy current analysis. IEEE Trans. on Magnetics, MAG-21:2231-2234, 1985.

[20] V. Girault and P. Raviart. Finite Element Approximation of the Navier-Stokes Equations. Lecture Notes in Math. \# 749, Springer-Verlag, New York, 1981. MR0548867 (83b:65122)

[21] P. Grisvard. Elliptic Problems in Nonsmooth Domains. Pitman, Boston, 1985. MR0775683 (86m:35044)

[22] R. Hiptmair. Finite elements in computational electromagnetism. Acta Numer., 11:237-339, 2002. MR2009375 (2004k:78028)

[23] J. D. Joannopoulos, R. D. Meade, and J. N. Winn. Photonic Crystals. Princeton University Press, Princeton NJ, 1995.

[24] A. Kameari. Three dimensional eddy current calculation using finite element method with a-v in conductor and $\omega$ in vacuum. IEEE Trans. on Magnetics, 24:118-121, 1988.

[25] T. Kato. Perturbation Theory for Linear Operators. Springer-Verlag, New York, 1976. MR0407617 (53 \#11389)

[26] A. Knyazev. Convergence rate estimates for iterative methods for a mesh symmetric eigenvalue problem. Sov. J. Num. Anal. Math. Modeling,, 2:371-396, 1987. MR0915330 (88i:65057)

[27] A. V. Knyazev. Preconditioned eigensolvers - an oxymoron? Electron. Trans. Numer. Anal., 7:104-123 (electronic), 1998. MR1667642(99h:65068)

[28] A. V. Knyazev. Toward the optimal preconditioned eigensolver: Locally optimal block preconditioned conjugate gradient method. SIAM J. Sci. Comput., 23(2):517-541, 2001. MR.1861263 (2003g:65050)

[29] F. Kukuchi. On a discrete compactness property for the nedelec finite elements. J. Fac. Sci. Univ. Tokyo, Sect. 1A, Math, 36:479-490, 1989.

[30] P. Leonard and D. Rodger. Finite element scheme for transient 3d eddy currents. IEEE Trans. on Magnetics, 24:58-66, 1988.

[31] P. Monk. A simple proof of convergence for an edge element discretization of maxwell's equations. Computational electromagnetics, Lect. Notes Comput. Sci. Eng., 28, Springer, Berlin, 2003. MR1986135 (2004i:78024) 
[32] P. Monk. Finite element methods for Maxwell's equations. Oxford Science Pub., Oxford, 2003. MR2059447(2005d:65003)

[33] P. Monk and L. Demkowicz. Discrete compactness and the approximation of Maxwell's equations in $\mathbb{R}^{3}$. Math. Comp., 70(234):507-523, 2001. MR.1709155 (2001g:65156)

[34] J. C. Nedelec. Mixed finite elements in $\mathbf{R}^{3}$. Numer. Math., 35:315-341, 1980. MR0592160 (81k:65125)

[35] J. C. Nedelec. A new family of mixed finite elements in $\mathbf{R}^{3}$. Numer. Math., 50:57-81, 1986. MR0864305 (88e:65145)

[36] I. Perugia, D. Schötzau, and P. Monk. Stabilized interior penalty methods for the timeharmonic Maxwell equations. Comput. Methods Appl. Mech. Engrg., 191(41-42):4675-4697, 2001. MR1929626 (2003j:78058)

[37] L. R. Scott and S. Zhang. Finite element interpolation of nonsmooth functions satisfying boundary conditions. Math. Comp., 54(190):483-493, 1990. MR.1011446 (90j:65021)

[38] C. M. Soukoulis. Photonic Band Gap Materials. Kluwer, Dordrecht, 1996.

Department of Mathematics, Texas A\&M University, College Station, Texas 778433368

E-mail address: bramble@math.tamu.edu

Department of Mathematics, Texas A\&M University, College Station, Texas 778433368

E-mail address: tkolev@math.tamu.edu

Department of Mathematics, Texas A\&M University, College Station, Texas 77843 3368

E-mail address: pasciak@math.tamu.edu 